\title{
Generalised conservation laws in non-local field theories
}

\author{
Alexander Kegeles ${ }^{1,2}$ and Daniele Oriti ${ }^{1}$ \\ 1 Max Planck Institute for Gravitational Physics (Albert Einstein Institute), \\ Am Mühlenberg 1, 14476 Potsdam-Golm, Germany, EU \\ alexander.kegeles@aei.mpg.de, daniele.oriti@aei.mpg.de \\ ${ }^{2}$ University of Potsdam Institute of Physics and Astronomy \\ Karl-Liebknecht-Strasse 24/25, \\ 14476 Potsdam-Golm, Germany, EU
}

\begin{abstract}
We propose a geometrical treatment of symmetries in non-local field theories, where the nonlocality is due to a lack of identification of field arguments in the action. We show that the existence of a symmetry of the action leads to a generalised conservation law, in which the usual conserved current acquires an additional non-local correction term, obtaining a generalisation of the standard Noether theorem. We illustrate the general formalism by discussing the specific physical example of complex scalar field theory of the type describing the hydrodynamic approximation of Bose-Einstein condensates. We expect our analysis and results to be of particular interest for the group field theory formulation of quantum gravity.
\end{abstract}

\section{INTRODUCTION}

The notion of symmetry is at the very foundation of our description of physical systems, in particular classical and quantum field theories. Symmetries characterise their physical content and interpretation. Their particle content is defined in terms of representations of the Poincaré group, and their classical and quantum dynamics is characterised e.g. by restrictions on the allowed field interactions. Symmetries are also one of the main tools to characterise their macroscopic properties, since different macroscopic phases of a given physical system are often efficiently characterised by symmetries and their (spontaneous) breaking. In fact, conservation laws following from symmetries may encode basically the whole dynamical content of many macroscopic systems, hydrodynamics being the obvious example. The corresponding universality class is largely independent of the microscopic details of the system, while the corresponding conserved charges identify key physical quantities. In local (quantum) field theories the connection between symmetries of the action and physically measurable quantities like conserved currents and charges is very well understood. This relation is given by the Noether theorem [1], that allows to identify and compute the conserved quantities and correspondent symmetry transformations.

The other main ingredient in our description of physical systems is indeed the notion of locality, which again largely dictates the structure of fundamental interactions and it is, both historically and conceptually, at the root of the very notion of fields.

Still, the necessity to go beyond the framework of local field theories has been voiced in several contexts. Non-local field theories are routinely used in condensed matter theory and many-body quantum theory, in the hydrodynamic approximation, e.g. in the theory of Bose-Einstein condensates [4] and even in relativistic theories [2, 3]. In this context, the field theory fails to be local in that the typical interaction term depends on more than a single spacetime point, i.e. the fields interact at a distance. Formally, this implies that the Lagrangian of the theory has a domain given by a direct product of several copies of the (spacetime) manifold on which fields are defined. However, the consequences of this non-local structure are rarely investigated in detail, mainly because such field theories are understood as the non-relativistic approximation of truly local field theories, and thus their non-local features are not considered of any fundamental significance.

More radical in scope are the proposals ${ }^{1}$ for non-local field theory descriptions of cosmological phenomena (see for example [6]) and of semi-classical black hole physics (see for example [7]). In all these cases the breakdown of strict locality even in spacetime-based physics is not understood as an artefact of the approximation used, but rather as the

\footnotetext{
${ }^{1}$ Notice that most such models are non-local in the sense that they involve an infinite number of field derivatives (see for example 5 |). It is unclear to us if there is a general connection between this type of non-locality and the one we deal with in our analysis. At any rate, we will not deal with this type of field theories.
} 
signal of an even more drastic departure from the local description of fundamental interactions at a deeper level of reality [8]. And locality is indeed the main feature of standard spacetime physics that is affected by effective models of quantum gravity which suggest a basis for quantum gravity phenomenology. Deformations of spacetime symmetries [9] and/or ideas from non-commutative geometry [10] are just few examples with fundamental non localities. In fact, several hints are accumulating that a more fundamental description of quantum gravity will imply a breakdown of the very notion of spacetime and its 'dissolution' at microscopic scales, only to emerge in a suitable continuum and semiclassical approximation from the collective dynamics of more fundamental, non-spatiotemporal degrees of freedom (see the discussion in [11], and references therein). The more fundamental quantum gravity description, in this perspective, will not be spacetime-based, by definition, and thus will not be 'local' in any usual sense. In itself this does not imply that a field theory framework cannot be devised for describing the fundamental degrees of freedom, even though it requires a more abstract type of field theories which obviously are not defined on a spacetime manifold. This more general class of field theories have been developed and go under the name of 'group field theories' [12].

Group field theories are quantum field theories defined on a group manifold, not to be identified with spacetime, but rather defining the dynamics of 'quanta of spacetime'. This formalism lies at the point of convergence of several quantum gravity approaches, being understood both as a second quantised reformulation of loop quantum gravity [13, 14] and as a generalisation and enrichment of tensor models [15] and simplicial gravity. Not being defined on spacetime, the standard type of locality has no reason to be invoked for these field theories. Indeed group field theories are non-local with respect to the group manifold that represents the domain of the fundamental fields. In fact, a peculiar non-local pairing of field arguments entering their interactions is a key characterising feature of the formalism. Moreover, a similar type of non-locality is present in a different type of field theories which lead to non-linear extensions of quantum cosmology [16], where the wave function interacts with itself non-locally on minisuperspace. The similarity is not entirely mysterious, given that the latter type of effective cosmological dynamics emerges from group field theories as the hydrodynamic approximation of the quantum dynamics of condensate states [17], and in a way which is completely analogous to what happens in real Bose-Einstein condensates. This type of quantum gravity models are our main motivation for considering the issue of symmetries and conservation laws in non-local field theories.

The issue is wide open. Indeed, in non-local field theories the standard Noether theorem fails and a general relation between symmetries and conservation laws is not known. For flat base manifolds and a special kind of non-local systems the connection between symmetries and conservation laws can be established [3, 18-21]. But a general treatment of the problem is lacking. And in the context of group field theories, a first, partial analysis was presented in [22].

Our goal, in this paper, is to provide a solid and general analysis of the connection between symmetries and conservation laws in non-local field theories, where the non-locality amounts to a lack of simple identification of field arguments in the Lagrangian.

We use differential geometrical language, in which the Lagrangian is seen as a function on many copies of a single first jet bundle, but the geometry of the base manifold of a single bundle is left unspecified [23]. This allows for a very general approach to the problem and leads to a result that can be applied to any non-local theory on curved space time in which the Lagrangian depends on first derivatives of the fields at most. This includes a large class of field theories used in condensed matter systems, and our motivating quantum gravity models, that is group field theories.

We derive the connection between symmetries and conserved quantities for non-local theories and obtain a Noetherlike conservation law augmented by a 'correction term' that results form the presence of non-locality. The resulting continuity equation is our main result and is what we call the 'generalised conservation law'. The result is rigorous and very general, for the class of theories it applies to but also because it concerns any continuous symmetry. Of course its main interest lies in the application to specific physical examples. In order to clarify and illustrate in detail our formalism, we present an explicit application of our result to the case of a field theory with 2-body interaction, as the one describing, say, the hydrodynamics of a Bose-Einstein condensate in the Gross-Pitaevskii approximation. We derive the generalised conservation laws, and show that the correction term admits an intuitive physical interpretation. The detailed application of our results to group field theories, on the other hand, will be presented elsewhere.

Our paper is structured as follows. An introduction of notation, conventions and the geometrical space that we use throughout the paper is presented in section II In section III we provide the definition of the type of non-local action we are concerned with, followed by the equations of motion and definition of symmetry transformations. We derive our main result, that is the generalised conservation law, in section IV. An application of our analysis to an explicit non-local field theory is presented in section $\mathrm{V}$. In the last section we briefly review the derivation of Ward identities in the functional integral formalism, and show the quantum counterpart of our main result. 


\section{NOTATION, CONVENTIONS AND BASIC DEFINITIONS}

In this section we introduce the notation, conventions and the main definitions of the geometrical space defining the framework for our analysis. In the concluding section of the paper, we will detail an explicit example of a non-local field theory analyzed with the methods developed in the bulk of the paper. We refer to the same example for further clarification of our notation and definitions.

\section{A. Vector and jet bundles}

A vector bundle $\pi: E \rightarrow M$ with the projection $\pi$, the total space $E$, the fiber $V$ and the base manifold $M$ is denoted simply with $E$. We also assume that the base manifold $M$ is orientable and is equipped with a (semi-)Riemannian metric. The covariant derivative is denoted with $D$ and the subscript $D_{q}$ denote the point at which the derivative is taken.

Capital greek letters will denote sections on $E$. In a local trivialization $U \subset M$, the section is given by $\Phi=\{q, \phi(q) \mid q \in U\}$. The correspondent functions $\phi: M \rightarrow V$ are called fields on $E$ and are denoted with the correspondent lower case greek letter. The space of smooth fields on $E$ is denoted by $\Gamma(E)$. Additional restrictions on this sets, such as local fields around $q \in M$, fields local in $U \subset M$ and compactly supported fields in $U \subset M$, are denoted by $\Gamma_{p}(E), \Gamma_{U}(E)$ and $\Gamma_{U, C}(E)$, respectively. ${ }^{2}$

The first jet bundle on $E$ is denoted $J b(E)$. Its fiber at point $q \in M$ is called the jet space $J b(E)_{q}=J_{q}$ and is isomorphic to the set of differentials of local fields around $q \in M$. Sections on $J b(E)$ are denoted by $j(\Phi)$ with correspondent fields $j(\phi) \simeq D \phi$ and are called prolonged section and fields, respectively. In a local trivialization, $j(\Phi)=\left\{\left(q, \phi(q), D_{q} \phi\right) \mid q \in U\right\}$.

\section{B. Geometrical construction}

The base manifold of $E$ is the domain of the Lagrangian, itself a functional of our physical fields. In local field theories it coincides with the domain of the fields (and of their derivatives). For example, in non-relativistic, Newtonian physics, and for a local field theory, the base manifold is a product of time and space as $M=\mathbb{R} \times \mathbb{R}^{3}$. In a more general situation (e.g. non-spacetime based theories like in group field theories), it can be a product of $N$ different manifolds $M=M^{1} \times \cdots \times M^{N}$. In theories corresponding to this more general situation, it is possible that the action and the Lagrangian are local on some sub-manifolds $M^{i}$ and non-local on the rest. Here by locality we mean that the arguments of the different fields (and their derivatives) appearing in the Lagrangian are identified with one another when they refer to such sub-manifolds $M^{i}$. An example of this situation is given again by non-relativistic field theories as used in condensed matter theory, where usually the fields appearing in the action differ by their spatial domain but are evaluated at the same time. We call this distribution of non-localities the combinatorial structure, and say that a theory is trivially non-local if its Lagrangian depends on $N$ fully distinct points. Local theories, in our setting, are those such that the fields appearing in the Lagrangian have all their arguments identified, thus effectively the Lagrangian depends on a single component $M$.

The underling jet bundle of a theory with trivial combinatorics is a product of $N$ jet bundles over $E$. It is again a vector bundle $E^{P} \simeq E^{\times N}$ with the base manifold $M^{P} \simeq M^{\times N}$ the fiber $V^{P} \simeq V^{\oplus N}$. The jet space at each point is a direct sum of jet spaces in the same way as it is the fibers $V^{P}$. That is for each $q \in M^{P} \simeq\left(q^{1}, \cdots, q^{N}\right)$ $J_{q}^{P} \simeq J_{q^{1}} \oplus \cdots \oplus J_{q^{N}}$

For a theory with non trivial combinatorial structure the underling base manifold $\mathcal{M}$ is a sub manifold of $M^{\times N}$. To be able to study this case, we assume that $\mathcal{M}$ can be isometrically embedded in $M^{\times N}$ by an embedding $f$ that consists of a combination of the following three maps:

The identity

$$
\begin{aligned}
\mathbb{1}: M & \rightarrow M \\
q & \mapsto q,
\end{aligned}
$$

\footnotetext{
${ }^{2}$ Even in local field theories it is well known, that solutions to variational problems might not be differentiable in general. In non local field theories the situations is even more complicated. In this paper we focus on the general procedure instead of treating the problem of existents of solutions. For this reason we are not distinguishing between smooth and $n$ times differentiable fields.
} 
the diagonal map

$$
\text { Di : } \begin{aligned}
M & \rightarrow M \times M \\
q & \mapsto(q, q),
\end{aligned}
$$

and the permutation map

$$
\text { Per : } \begin{aligned}
M^{1} \times M^{2} & \rightarrow M^{2} \times M^{1} \\
\left(q^{1}, q^{2}\right) & \mapsto\left(q^{2}, q^{1}\right) .
\end{aligned}
$$

For example for the mentioned class of theories, which are local in time and non-local in space, we get the embedding $\operatorname{map} f: \mathbb{R} \times \mathbb{R}^{3} \times \mathbb{R}^{3} \hookrightarrow \mathbb{R} \times \mathbb{R}^{3} \times \mathbb{R} \times \mathbb{R}^{3}$ by setting

$$
f=(\mathbb{1} \times \operatorname{Per} \times \mathbb{1})(\operatorname{Di} \times \mathbb{1} \times \mathbb{1}) .
$$

The space $\mathcal{M}$ is therefore connected to $M^{P}$ by the embedding $f$. We define our vector bundle $\mathcal{E}$ for the general case as the pull-back vector bundle of $E^{\times N}$. That is,

$$
\mathcal{E}:=f^{*} E^{\times N}
$$

The jet space at each point $q \in \mathcal{M}$ is $\mathcal{J}_{q}=\left\{\mathcal{D}_{q} \vec{\phi}=\left(D_{q^{1}} \phi^{1}, \cdots, D_{q^{N}} \phi^{N}\right) \mid \phi \in \Gamma_{q}\left(E^{P}\right)\right\}$ where $\mathcal{D}$ denotes the covariant derivative on $\mathcal{M}$ given by the pull back of $D^{\times N}$ and $q^{i}=\operatorname{pr}^{i}(f(q))$.

The canonical volume form on $M$ is denoted vol. In local coordinates it has the usual expression vol $=\sqrt{|g|} \mathrm{d} x^{1} \wedge$ $\cdots \mathrm{d} x^{I}$. The volume form on $\mathcal{M}$ is denoted by $\mathrm{Vol}=f^{*} \operatorname{vol}^{\times N}$. We will also sometimes sloppy denote $\operatorname{vol}_{q}\left(\operatorname{Vol}_{\bar{q}}\right)$ to underline that the integration is (is not) performed over the manifold whose points we label $q$.

In cases where we need to use charts we denote the dimension of the base manifold and the fiber dim $(M)=\mathrm{d}_{M}$ and $\operatorname{dim}(V)=\mathrm{d}_{V}$, respectively.

We call $\mathfrak{D}^{i}$ the domain of the field $\phi^{i}$ and symbolically use the notation of the delta $\delta^{i}(q)$ under the integral referring to the following identity

$$
\int_{\mathcal{M}} \delta^{i}(q) f \mathrm{Vol}:=\int_{\mathcal{M} \backslash \mathfrak{D}^{i}} f \mathrm{Vol}_{\mathfrak{D}^{i}}
$$

and the domain $\mathfrak{D}^{i}$ is set to $q \in M$.

We will derive functions with respect to a parameter $\epsilon$. In this case we always assume that the derivative is taken at the point $\epsilon=0$ and don't write it explicitly to simplify readability. In the single case where the derivative is not assumed to be at the point zero we will explicitly denote it.

\section{Fiber derivatives}

It will be very useful to use notation that allows to avoid indices and still suggests contractions of vector fields in the natural way. In short, we treat derivatives as 1-forms on the appropriate space. This allows us to write contractions as dual pairings without referring to the index notation, improving readability.

Treating the Lagrangian $L$ as a function on three spaces - the base manifold, the fiber and the jet - we introduce the common notions of derivatives on each of these spaces.

Assuming that the points of the fiber $\mathcal{V}$ and the jet $\mathcal{J}$ are given by a smooth field $\vec{\phi} \in \Gamma(\mathcal{E})$ we can treat the Lagrangian as a function $L: \mathcal{M} \rightarrow \mathbb{R}$. Its derivative at a point $q \in \mathcal{M}$ is then a function

$$
\mathcal{D}_{q} L: T_{q} \mathcal{M} \rightarrow \mathbb{R}
$$

In other words it is a one form on the tangent bundle $T \mathcal{M}$.

On the other hand fixing the base point $q \in \mathcal{M}$ we can treat the Lagrangian as a function $L: \mathcal{E}_{q} \times \mathcal{J}_{q} \rightarrow \mathbb{R}$.

The derivative of $L$ in the fiber $\mathcal{V}$ is denoted by

$$
\left.\mathcal{D}_{\mathcal{V}} L\right|_{\Phi(q)}: T_{\Phi(q)} \mathcal{E}_{q} \rightarrow \mathbb{R}
$$

It is seen as a 1 -form on $T_{\Phi(q)} \mathcal{E}_{q} \simeq \mathcal{E}_{q}$. We will often abandon labelling the base point and write $\mathcal{D}_{\mathcal{V}} L(\vec{\phi})$ for $\vec{\phi} \in \Gamma(\mathcal{E})$, meaning the 1 -form $\mathcal{D}_{\mathcal{V}} L$ contracted with the vector $\vec{\phi}(q) \in \mathcal{E}_{q}$. In local coordinates $\left(x^{i}, u^{j}, u_{x^{i}}^{j}\right)_{n}$, for $i \in\left\{1, \cdots \mathrm{d}_{M}\right\}$ 
$j \in\left\{1, \cdots, \mathrm{d}_{V}\right\}, n \in\{1, \cdots N\}$, and with $x_{n}^{j}=x_{n}^{j}(q), u^{j, n}=\phi^{j n}\left(x_{1}^{j}, \cdots, x_{N}^{j}\right)$ and $u_{x_{n}^{i}}^{j, n}=D_{x_{n}^{i}} \phi^{j n}\left(x_{1}^{j}, \cdots x_{N}^{j}\right)$, we get

$$
\mathcal{D}_{\mathcal{V}} L(\phi)=\sum_{n=1}^{N} \sum_{s=1}^{\mathrm{d}_{V}} \partial_{u^{s, n}} L\left(\left(x, u, u_{x}\right)\right) u^{s, n}
$$

It follows directly from the above coordinate representation that the fiber derivative splits in a natural way into contractions on each $E$ as

$$
\mathcal{D}_{\mathcal{V}} L(\vec{\phi})=D_{V^{1}} L\left(\phi^{1}\right)+\cdots+D_{V^{n}} L\left(\phi^{n}\right)
$$

The derivative of $L$ in the jet $\mathcal{J}$ is denoted by

$$
\mathcal{D}_{\mathcal{J}} L: T_{j(\Phi)} \mathcal{J}_{q} \rightarrow \mathbb{R}
$$

again with $T_{j(\Phi)} \mathcal{J}_{q} \simeq \mathcal{J}_{q}$. It is a function on the differentials of fields $\mathcal{D}_{q} \vec{\phi}$. The later, however, are linear functions on $T_{q} \mathcal{M}$ which are isomorphic to sections of the product bundle $E \otimes(T \mathcal{M})^{*}$. For this reason $\mathcal{D}_{\mathcal{J}} L$ is isomorphic to sections of $E^{*} \otimes T \mathcal{M}$. We mean exactly this natural contraction when we write $\mathcal{D}_{\mathcal{J}} L\left(\mathcal{D}_{q} \vec{\phi}\right)$. In coordinates, we can explicitly write

$$
\mathcal{D}_{\mathcal{J}} L(D \phi)=\sum_{n=1}^{N} \sum_{s=1}^{\mathrm{d}_{V}} \sum_{i=1}^{\mathrm{d}_{M}} \partial_{u_{x_{n}^{i}}^{s, n}} L\left(\left(x, u, u_{x}\right)\right) \cdot u_{x_{n}^{i}}^{s, n}
$$

This contraction splits in the same way as above in

$$
\mathcal{D}_{\mathcal{J}} L(D \phi)=D_{J^{1}} L\left(D \phi^{1}\right)+\cdots+D_{J^{n}} L\left(D \phi^{n}\right)
$$

It will be useful to integrate by parts the above relation to get

$$
\mathcal{D}_{\mathcal{J}} L(D \vec{\phi})=\operatorname{tr}\left[\mathcal{D}\left(\mathcal{D}_{\mathcal{J}} L\right)\right](\vec{\phi})-\operatorname{tr}\left[\mathcal{D}\left(\mathcal{D}_{\mathcal{J}} L(\vec{\phi})\right)\right]
$$

where the trace is understood as the contraction of the 1 -form coming from $\mathcal{D}$ with the vector part $T_{p} \mathcal{M}$ of the $\mathcal{D}_{\mathcal{J}} L$. In local coordinates the trace becomes

$$
\operatorname{tr}\left[\mathcal{D}\left(\mathcal{D}_{\mathcal{J}} L(\vec{\phi})\right)\right]=\sum_{n=1}^{N} \sum_{i=1}^{\mathrm{d}_{M}} \sum_{j=1}^{\mathrm{d}_{V}} \partial_{x_{n}^{i}} \partial_{u_{x_{n}^{i}}^{j, n}} L \cdot u^{j, n}
$$

which is the covariant definition of divergence. We write

$$
\mathcal{D}_{\mathcal{J}} L\left(\mathcal{D}_{q} \vec{\phi}\right)=\operatorname{div}\left[\mathcal{D}_{\mathcal{J}} L\right](\vec{\phi})-\operatorname{div}\left[\left(\mathcal{D}_{\mathcal{J}} L(\phi)\right)\right]
$$

Note that also the right-hand-side has a natural splitting into fibers as

$$
\operatorname{div}\left(\mathcal{D}_{\mathcal{J}} L\right)(\vec{\phi})=\operatorname{div}_{\mathfrak{D}^{1}}\left[D_{J^{1}} L\right]\left(\phi^{1}\right)+\cdots+\operatorname{div}_{\mathfrak{D}^{N}}\left[D_{J^{n}} L\right]\left(\phi^{n}\right)
$$

where we denote $\operatorname{div}_{\mathfrak{D}^{i}}$ the divergence on the single domain of $\phi^{i}$. As we can see the fiber derivatives split in a natural way into a sum indexed by the field index $j \in\{1, \cdots, N\}$. However, notice that the divergence of a general vector field $X \in T \mathcal{M}$ does not split in the above way.

To conclude the section we present a short table (II) of symbols that we use throughout the paper. 


\begin{tabular}{c|l|cl}
\multicolumn{2}{|l|}{ Single vector bundle } & \multicolumn{2}{c}{ Pull back bundle } \\
\hline$E$ & Total space & $\mathcal{E}$ & Total space \\
\hline$M$ & Base manifold & $\mathcal{M}$ & Base manifold \\
\hline$V$ & Single fiber & $\pi^{\mathcal{E}}$ & Projection on $\mathcal{M}$ \\
\hline$\pi$ & Projection on $M$ & $\mathcal{J}$ & Jet space \\
\hline$J$ & Jet space & $\mathcal{J} b(\mathcal{E})$ & Jet bundle \\
\hline$J b(E)$ & Jet bundle & $\mathcal{D}$ & Covariant derivative \\
\hline$D$ & Covariant derivative & $\mathcal{D}_{\mathcal{V}}$ & Fiber derivative in $\mathcal{V}$ \\
\hline$D_{V}$ & Fiver derivative in $V$ & $\mathcal{D}_{\mathcal{J}}$ & Fiber derivative in $\mathcal{J}$ \\
\hline$D_{J}$ & Fiber derivative in $J$ & $\operatorname{Vol}$ & Volume element on $\mathcal{M}$ \\
\hline vol & Volume element $M$ & $\mathfrak{D}^{i}$ & Domain of the fiber $V^{i} \subset \mathcal{V}$ \\
\hline & & $\mathrm{d}_{\mathcal{M}}$ & Dimension of $\mathcal{M}$ \\
\hline $\mathrm{d}_{M}$ & Dimension of $M$ & $\mathrm{~N}_{V}$ & Number of non local points \\
\hline $\mathrm{d}_{V}$ & Dimension of $V$ & $\vec{\phi}$ & Smooth fields in $\Gamma(\mathcal{E})$ \\
\hline$\phi$ & Smooth fields in $\Gamma(E)$ &
\end{tabular}

Table I: An overview of the most used symbols in this paper

\section{SYMMETRIES AND CONTINUITY EQUATIONS FOR NON LOCAL ACTIONS}

In this section we give the definition of the non-local field theories, described by non-local action functionals, that we consider in this paper. Loosely speaking, we call non-local an action functional that is defined by a Lagrangian which depends on $N$ copies of a jet bundle. Theories described by this kind of actions often appear when the physical system consists of many particles, their interaction can not be neglected and is not of contact type, in the classical limit. They appear in various branches of physics such as hydrodynamics, condensed matter and solid state physics. In fact, an example of non-local theories of the above type is given by effective theories, which arise when one disregards some microscopic degrees of freedom to obtain a theory of fewer variables. The price to pay is often the emergence of non-locality of the type we study. The most prominent examples of this type are models with Coulomb interaction, which are effective theories of an underlying local (and relativistic) electrodynamics. They also appear in some models of quantum gravity, although of course the physical interpretation is very different.

We begin with the definition of a non-local geometrical Lagrangian and action, as the most natural extension of the usual local ones [23, 24]. From them, we obtain what we call the physical non-local Lagrangians. In the following two subsections we then apply the variational principle and the theory of Lie groups to obtain the equations of motions and local symmetry statements.

\section{A. The non local action}

In this subsection we introduces the various definitions for the non-local geometrical and physical actions. We will make a distinction between geometrical and physical quantities with an superscript $G$ or $P$ respectively. The motivation for this separation will become apparent at the end of this section. We begin with the definition of non local geometrical Lagrangian.

Definition 1. Let $\mathcal{J} b(\mathcal{E})$ be a jet bundle over the vector bundle $\mathcal{E}$. A map $L^{G}: \mathcal{J} b(\mathcal{E}) \rightarrow \mathbb{R}$ is called a geometrical Lagrangian if it is differentiable in $\mathcal{M}, \mathcal{V}, \mathcal{J}$ with respect to our definitions of fiber derivatives in the previous section.

Recall that the fiber $\mathcal{V}$ is isomorphic to the direct sum of $N$ fibers $V$. We call $N$ the degree of non locality, with $N=1$ defining a local theory. Just as in the local case we can define the non-local geometrical action by integrating the Lagrangian over a region of the base manifold.

Definition 2. Let $\Omega \subset \mathcal{M}$ be an open region. A non-local geometrical action is a functional $S_{\Omega}^{G}: \Gamma_{\Omega}(\mathcal{E}) \rightarrow \mathbb{R}$ defined 
by the Lagrangian as follows

$$
S_{\Omega}^{G}[\vec{\phi}]=\int_{\Omega} L^{G}(j(\Phi)) \text { Vol. }
$$

Since a field at each point $\mathcal{E}$ has a direct sum structure $\vec{\phi}(q)=\left(\phi^{1}, \cdots, \phi^{N}\right)(f(q))$ the above action corresponds to a situation where the fields cary labels, which makes them distinguishable. In this case different fields are evaluated at different points. Such theories are called colored sometimes. However, in our analysis we are mainly interested in non-local physical systems with a single field.

To define the Lagrangian on a single field we use the diagonal map

$$
\begin{aligned}
& \imath_{N}: \Gamma(E) \rightarrow \bigoplus_{i=1}^{N} \Gamma(E) \simeq \Gamma(\mathcal{E}) \\
& \phi \quad \mapsto(\phi, \cdots, \phi),
\end{aligned}
$$

which can be seen as an embedding of $\Gamma(E)$ into the larger space $\Gamma(\mathcal{E})$. We will sometimes write $\imath_{N}(\phi)=\sum_{i}^{N} \phi \otimes e_{i}$ with the standard basis $\left\{e_{i}\right\}$ in $\mathbb{R}^{N}$. Finally we define the non local physical Lagrangian as follows.

Definition 3. Let $L^{G}$ be a geometrical Lagrangian on $\mathcal{J} b(\mathcal{E})$ and $\imath_{N}$ be the diagonal embedding map. The corresponding non-local physical Lagrangian $L^{P}: \mathcal{M} \times \Gamma(E) \rightarrow \mathbb{R}$ is defined point-wise by

$$
L^{P}(q, \phi)=L^{G}\left(q,\left[\imath_{N} \phi\right](q), \mathcal{D}_{q}\left[\imath_{N} \phi\right]\right)
$$

It is the physical Lagrangian which has a more straightforward physical interpretation (hence the chosen name), even though the geometrical interpretation is much clearer by the geometrical Lagrangian. Because of this, we will first reformulate the physical conditions such as equations of motion in terms of the geometrical Lagrangian and use then the well established theory of Lie groups to obtain local symmetry conditions.

First, we introduce the non-local physical action, whose variation will lead to the equations of motion.

Definition 4. Let $U \subset M$ be open, and $\Omega=f^{-1}\left(U^{\times N}\right) \subset \mathcal{M}$. A non-local physical action is a functional $S_{\Omega}^{P}: \Gamma_{U}(E) \rightarrow \mathbb{R}$ defined by the physical Lagrangian as

$$
S_{\Omega}^{P}[\phi]=\int_{\Omega} L^{P}(\cdot, \phi) \mathrm{Vol}
$$

Using the definition of the physical Lagrangian and the geometrical action we see the connection between the physical and geometrical quantities

$$
S_{\Omega}^{P}=S_{\Omega}^{G} \circ \imath_{N}
$$

In this formulation we can state that the main formal reason for the failure of the standard Noether theorem in the non-local case is the difference between the physical and geometrical action, as we will see shortly. Notice that, indeed, in the local case $(N=1)$ the inclusion map $\imath_{N}$ is the identity, which also clarifies why the connection of geometrical symmetries and physical equations of motion is natural. In the non-local case, on the other hand, we will be facing a problem in connecting the equations of motion to the symmetry properties of the geometrical functional.

In the next section we present the equations of motion in terms of the geometrical action and Lagrangian.

\section{B. Non local equations of motion}

In this section we apply the variational principle to the non-local physical action $S^{P}$ and obtain the (semi-local) equations of motion.

Lemma 1. Let $\varphi_{\epsilon} \in \Gamma(E)$ be a family of smooth fields, differentiable in the parameter $\epsilon$. The diagonal inclusion map $\imath_{N}$ commutes with the partial derivative in $\epsilon$ in the following sense,

$$
\partial_{\epsilon} \imath_{N}\left(\varphi_{\epsilon}\right)=\imath_{N} \partial_{\epsilon}\left(\varphi_{\epsilon}\right) .
$$

Proof. By direct computation we obtain

$$
\partial_{\epsilon} \imath_{N}\left(\varphi_{\epsilon}\right)=\partial_{\epsilon}\left(\varphi_{\epsilon}, \cdots, \varphi_{\epsilon}\right)=\left(\partial_{\epsilon} \varphi_{\epsilon}, \cdots, \partial_{\epsilon} \varphi_{\epsilon}\right)=\imath_{N}\left(\partial_{\epsilon} \varphi_{\epsilon}\right) .
$$


Let $\varphi \in \Gamma_{U, C}(E)$ be an arbitrary, compactly supported, smooth field in $U$ and let $\Omega \subset \mathcal{M}$ such that $f(\Omega)=U^{\times N}$. The variation of $S_{\Omega}^{P}$ is given by

$$
\begin{aligned}
\left.\mathrm{d} S^{P}\right|_{\phi}(\varphi) & =\partial_{\epsilon} S_{\Omega}^{P}(\phi+\epsilon \varphi) \\
& =\int_{\Omega} \partial_{\epsilon} L^{P}(\cdot, \phi+\epsilon \varphi) \mathrm{Vol} \\
& =\int_{\Omega} \partial_{\epsilon} L^{G}\left(\cdot, \imath_{N}[\phi+\epsilon \varphi], \mathcal{D}\left(\imath_{N}[\phi+\epsilon \varphi]\right)\right) \text { Vol } .
\end{aligned}
$$

By lemma 1 we get

$$
\left.\mathrm{d} S^{P}\right|_{\phi}(\varphi)=\left.\int_{\Omega} \mathcal{D}_{\mathcal{V}} L^{G}\right|_{j\left(\Phi_{D}^{P}\right)}\left[\imath_{N} \varphi\right]+\left.\mathcal{D}_{\mathcal{J}} L^{G}\right|_{j\left(\Phi^{P}\right)}\left[\mathcal{D}\left(\imath_{N} \varphi\right)\right] \text { Vol }
$$

And by partial integration we obtain

$$
\left.\mathrm{d} S^{P}\right|_{\phi}(\varphi)=\int_{\Omega}\left[\mathcal{D}_{\mathcal{V}} L^{G}-\operatorname{div}\left(\mathcal{D}_{\mathcal{J}} L^{G}\right)\right]_{j\left(\Phi^{P}\right)}\left[\imath_{N} \varphi\right] \text { Vol } .
$$

The right-hand-side defines the Euler-Lagrange equations by the extremality condition

$$
\left.\mathrm{d} S\right|_{\phi}(\varphi)=0 \quad \forall \varphi \in \Gamma_{U, C}(E),
$$

We define the Euler 1-form as $E:=\left[\mathcal{D}_{\mathcal{V}} L^{G}-\operatorname{div}\left(\mathcal{D}_{\mathcal{J}} L^{G}\right)\right]$ (not to be confused with the vector bundle $E$ ), and write

$$
\left.\mathrm{d} S\right|_{\phi}(\varphi)=\int_{\Omega} E_{\left(\Phi^{P}\right)} \circ \imath_{N}(\varphi) \text { Vol. }
$$

In the following we will abandon the label for the base point as well as the notation of the integral domain $\Omega$ and simply write $\mathrm{d} S(\varphi)=\int_{\Omega} E \circ \imath_{N}(\varphi)$. This equation gives rise to semi local equations of motion by the fundamental lemma of variations, that we state below for convenience (for further references see for example [25]).

Lemma 2 (Fundamental lemma of variation). Let $f$ be a continuous, real-valued function on some region $U \subset \mathbb{R}^{m}$, and suppose that

$$
\int_{U} f(x) \varphi(x) d x=0
$$

holds for all $\varphi \in C_{C}^{\infty}(U)$ with $\varphi \geq 0$. Then

$$
f(x)=0
$$

for all $x \in U$.

A corollary of this lemma provides the semi-local equations of motion.

Corollary 1. Let $E$ be a one form on $\mathcal{E}$. Then we can locally write $E=\sum_{i=1}^{N} f^{i, j} \otimes v^{j} \otimes e^{i}$ with continues functions $f^{i, j}$ on $\Omega \times \mathcal{V}$, one forms $v^{j}$ on $V$ and dual vectors $e^{i}$ given by $e^{i}\left(e_{j}\right)=\delta_{i, j}$ for the standard basis $\left\{e_{j}\right\}_{j=1 \cdots N}$ in $\mathbb{R}^{N}$. If

$$
\int_{\Omega}\left(E \circ \imath_{N}\right)(\varphi) V o l=0
$$

for all $\varphi \in \mathcal{C}_{\mathcal{C}}^{\infty}(U)$. Then we have for all $q \in U$

$$
\int_{\Omega \backslash \mathfrak{D}^{1}} f^{1} \operatorname{Vol}_{\overline{\mathfrak{D}}^{1}}+\cdots+\int_{\Omega \backslash \mathfrak{D}^{N}} f^{N} \operatorname{Vol}_{\mathfrak{D}^{-}}=0
$$

where the domain over which the integration is not performed is set to q. 
Proof. Applying the definitions we get

$$
\begin{aligned}
\int_{U^{\times N}} E \circ \imath_{N}(\varphi) \mathrm{Vol} & =\int_{\Omega} E\left[\imath_{N}(\varphi)\right] \mathrm{Vol} \\
& =\sum_{j=1}^{v} \sum_{i=1}^{n} \int_{\Omega}\left(f^{i, j} \otimes v^{j} \otimes e^{i}\right)\left[\varphi^{j} \otimes v_{j} \otimes e^{i}\right] \\
& =\sum_{j=1}^{v} \int_{\Omega}\left(f^{1, j} \cdot \varphi^{j}+\cdots+f^{n, j} \cdot \varphi^{j}\right) \mathrm{Vol} \\
& =\sum_{j=1}^{v} \int_{U}\left\{\sum_{i=1}^{n} \int_{\Omega \backslash \mathfrak{D}^{i}} f^{i, j} \operatorname{Vol}_{\overline{\mathfrak{D}}^{i}}\right\} \varphi^{j} \mathrm{vol} .
\end{aligned}
$$

By assumption, the functions $f^{j}:=\left\{\sum_{i=1}^{n} \int_{U^{\times N-1}} f^{i, j} \mathrm{Vol}\right\}$ are continuous on $U$. Since $\varphi^{j}$ are independent for different $j$ the statement follows from the fundamental lemma.

The semi-local equations of motion become

$$
E L[\cdot](q)=\sum_{i}^{N} \int_{\Omega \backslash \mathfrak{D}^{i}} E^{i}(q) \circ\left[\imath_{N} \cdot\right] \mathrm{Vol},
$$

with the 1-forms $E^{i}=\left[D_{V^{i}} L^{G}-\operatorname{div}_{i}\left(D_{J^{i}} L^{G}\right)\right]$. Here the point $q \in M$ is subsequently identified with each domain $\mathfrak{D}^{i}$ on which the integration is not performed. Notice that these equations are local in $q \in M$ but also depend on the value of the field at other points on $U$ (which follows from the non-local nature of the action). For this reason we call them 'semi-local'.

For the sake of readability we will write

$$
E L[\cdot](q)=\sum_{i=1}^{N} \int_{\Omega} \delta^{i}(q) E^{i}\left[\imath_{N} \cdot\right] \mathrm{Vol},
$$

using the delta notation introduced earlier. Note that due to the above description $E L$ is a 1-form on the fiber of $\mathcal{E}$, or equivalently on vertical vector fields. In the local case $N=1$ which implies $\imath_{N}=\mathrm{id}$ and $\Omega=U$, the sum vanishes and we obtain the local equations of motion

$$
E L[\cdot](q)=E[\cdot](q)=\left\{D_{V} L-\operatorname{div}\left[D_{J} L\right]\right\}_{\Phi(q)}[\cdot] .
$$

Let us conclude this section with a couple of additional remarks.

- The domain of integration for the physical action is chosen to be $f^{-1}\left(U^{\times N}\right)$. This is important since the case with a general $\Omega \subset \mathcal{M}$ generates additional technical problems, which we do not address here. The solution space of the theory gets additional restrictions on the boundaries of each $\Omega$. In a simple case $f=\mathbb{1}$ and for $\Omega=U^{1} \times U^{2} \times \cdots \times U^{N}$ with $U^{1} \subset U^{2} \subset \cdots U^{N}$ the variation of the action has to happen in the direction of smooth fields $\eta$ that are compactly supported on all $U^{i}$ in $\Omega$. That implies that $\eta$ has to vanish on at least $N$ open sets inside $U^{N}$. This condition would affect the subsequent analysis.

- In a non-local theory as we have defined it, adding to the Lagrangian the total divergence on $\mathcal{M}$ of a suitable tuple function $P$ can change the equations of motion and therefore lead to a non-equivalent Lagrangian. This can be easily seen for the case $f=\mathbb{1}$. Due to Stokes' theorem, the action will get an additional term which depends only on the boundary of $\Omega=U^{\times N}$. However, the variation is not assumed to vanish on the boundary of $\Omega$ but just on the boundary of $U$. The boundary of $\Omega$ is instead proportional to $\partial U^{\times N} \simeq \partial U \times U^{N-1}$, while the test field $\eta$ is vanishing only on $\partial U$, and therefore the added function $P$ is affected by the variation. This feature, which may lead to complications, distinguishes in general the non-local case from the local one.

\section{Symmetry of the non local action}

In this section we define the notion of a symmetry for the non-local geometrical action. This notion is global, meaning that it is a statement about integral quantities. However, we show that this notion is equivalent to a fully local condition in exactly the same way as it is in the local case. 
A vector field $X \in T \mathcal{E}$ induces an action of a one parameter group $G$ on $\mathcal{E}$ by translating the points on $\mathcal{E}$ along the flow $c_{\epsilon}$ of $X$. The vector field $X$ is then homomorphic to the Lie algebra $\mathfrak{g}$ of the group $G$ and is called the infinitesimal generator of the group action. Specifically, let · denote the action of the group $G$ on $\mathcal{E}$. Then for a group element $g_{\epsilon}=\exp (\epsilon v)$ with $v \in \mathfrak{g}$ and $\Phi(q)$ a point on $\mathcal{E}$ the corresponding vector field is

$$
\partial_{\epsilon} g_{\epsilon} \cdot \Phi(q)=X_{\Phi(q)}=\partial_{\epsilon} c_{\epsilon}(\Phi(q))
$$

where $c_{\epsilon}$ denotes the flow of $X$ on $\mathcal{E}$.

By the bundle projection $\pi^{\mathcal{E}}$ we can split every vector field $X$ as $X=X_{\mathcal{M}}+X_{\mathcal{V}}$ where $X_{\mathcal{M}}=\mathrm{d} \pi^{\mathcal{E}}(X)$ and $X_{\mathcal{V}}=X-X_{\mathcal{M}}$. At each point the vector field $X_{\mathcal{M}}$ is tangent to the base manifold and $X_{\mathcal{V}}$ is parallel to the fiber. We call the corresponding flows $c_{\mathcal{M} \epsilon}$ and $c_{\mathcal{V}}$, respectively. At every point we get

$$
\partial_{\epsilon} c_{\epsilon}=X=X_{\mathcal{M}}+X_{\mathcal{V}}=\partial_{\epsilon} c_{\mathcal{M} \epsilon}+\partial_{\epsilon} c_{\mathcal{V} \epsilon}=\partial_{\epsilon}\left(c_{\mathcal{M} \epsilon}, c_{\mathcal{V} \epsilon}\right)
$$

Integrating from 0 to $\epsilon$ and using $c_{0}=c_{\mathcal{M} 0}=c_{\mathcal{V} 0}=\mathbb{1}$ we get locally

$$
c_{\epsilon}=\left(c_{\mathcal{M} \epsilon}, c_{\mathcal{V} \epsilon}\right)
$$

Notice that $c_{\mathcal{M} \epsilon}: \mathcal{E} \rightarrow \mathcal{M}$ and $c_{\mathcal{E} \epsilon}: \mathcal{E} \rightarrow \mathcal{V}$. However, fixing a section $\Phi$ allows us to view the transformation maps as

$$
c_{\mathcal{M} \epsilon}: \mathcal{M} \rightarrow \mathcal{M} \quad c_{\mathcal{E} \epsilon}: \mathcal{M} \rightarrow \mathcal{V} .
$$

Therefore, locally for any $\Phi(q)=(q, \vec{\phi}(q))$ and any fixed $\vec{\phi} \in \Gamma(\mathcal{E})$ the group action can be split as

$$
c_{\epsilon}(q, \vec{\phi}(q))=\left(c_{\mathcal{M} \epsilon}(q), c_{\mathcal{V} \epsilon} \circ \vec{\phi} \circ c_{\mathcal{M} \epsilon}^{-1} \circ c_{\mathcal{M} \epsilon}(q)\right) .
$$

Calling the new points $c_{\mathcal{M} \epsilon}(q)=q_{\epsilon}$ and the transformed fields as $\vec{\phi}_{\epsilon}:=c_{\mathcal{V} \epsilon} \circ \vec{\phi} \circ c_{\mathcal{M} \epsilon}^{-1}$ we obtain the transformation of the section as

$$
\Phi_{\epsilon}\left(q_{\epsilon}\right)=\left(q_{\epsilon}, \vec{\phi}_{\epsilon}\left(q_{\epsilon}\right)\right)
$$

The transformation of $\vec{\phi}$ induce a transformation of the prolonged fields $j(\vec{\phi})$, which we call $c_{J \epsilon}$, given by

$$
c_{J \epsilon} \circ j(\vec{\phi}) \circ c_{\mathcal{M} \epsilon}^{-1}=j\left(c_{\mathcal{V} \epsilon} \circ \vec{\phi} \circ c_{\mathcal{M} \epsilon}^{-1}\right)=j\left(\vec{\phi}_{\epsilon}\right)
$$

We note that the section $\Phi_{\epsilon}$ can be treated as a graph on the transformed manifold as in Eq. 6 or equivalently as a function on the non-transformed base manifold as

$$
\Phi_{\epsilon}(q)=\left(c_{\mathcal{M} \epsilon}(q), \vec{\phi}_{\epsilon} \circ c_{\mathcal{M} \epsilon}(q)\right) .
$$

The section of the jet bundle becomes

$$
j\left(\Phi_{\epsilon}\right)(q)=\left(c_{\mathcal{M} \epsilon}, \vec{\phi}_{\epsilon} \circ c_{\mathcal{M} \epsilon}, j\left(\vec{\phi}_{\epsilon}\right) \circ c_{\mathcal{M} \epsilon}\right)(q) .
$$

The variation of the action along $X$ is then given by

$$
\begin{aligned}
\left.\mathrm{d} S\right|_{\phi^{P}}(X)=\partial_{\epsilon} \tilde{S}\left[\vec{\phi}_{\epsilon}\right] & =\partial_{\epsilon} \int_{c_{\mathcal{M} \epsilon}(\Omega)} L\left(j\left(\Phi_{\epsilon}\right)\right) \mathrm{Vol} \\
& =\int_{\Omega} \partial_{\epsilon}\left\{L\left(j\left(\Phi_{\epsilon}\right)\right) c_{M \epsilon}^{*} \mathrm{Vol}\right\} .
\end{aligned}
$$

It turns out to be very convenient to define the characteristic vector field of $X$ as

$$
X_{Q}:=\partial_{\epsilon} \vec{\phi}_{\epsilon}=X_{\mathcal{V}}-\mathcal{D} \vec{\phi}\left(X_{\mathcal{M}}\right),
$$

and call the coefficient of $X_{Q}$ the characteristic $Q$ of $X$. Notice that $X_{Q}$ is parallel to the fiber, since

$$
\mathrm{d} \pi^{\mathcal{E}}\left(X_{Q}\right)=0
$$


Definition 5. A local group $G$ acting on $\mathcal{E}$ is called a symmetry group of the non-local action $S_{\Omega}$, if a transformation $c \in G$, written in local trivialization as $\left(c_{\mathcal{M}}, c_{\mathcal{V}}\right)$, satisfies

$$
\tilde{S}(c \circ \vec{\phi}):=\int_{c_{\mathcal{M}}(\Omega)} L(c \circ j(\Phi)) \mathrm{Vol}=\int_{\Omega} L(j(\Phi)) \mathrm{Vol}=S(\vec{\phi})
$$

We now adopt the well known theorem that connects the symmetry transformation to a local change of the Lagrangian. The mere difference of the local case to our situation is that the vector bundle in the local case is a single $E$ whereas in our case it is $\mathcal{E}$. It is therefore obvious that geometrically these two cases are not different which is why the theorem caries over one to one. Nevertheless, we present the theorem as well as its prove for the convince of the reader to adopt it to our notation. The proof below follows closely the proof in [23] and partially in [24].

Theorem 1. A connected one-parameter group $G$ of transformations acting on $\mathcal{E}$ is a symmetry group of the action $S$ if and only if

$$
\left[\mathcal{D} L\left(X_{\mathcal{M}}\right)+\mathcal{D}_{\mathcal{V}} L\left(X_{Q}\right)+\mathcal{D}_{\mathcal{J}} L\left(D X_{Q}\right)+L \operatorname{div}\left(X_{\mathcal{M}}\right)\right]=0
$$

whereX is the infinitesimal generator of the group.

Proof. If $c_{\epsilon}=\left(c_{\mathcal{M} \epsilon}, c_{\mathcal{V} \epsilon}\right)$ is a symmetry transformation then

$$
\partial_{\epsilon} \tilde{S}\left(\phi_{\epsilon}\right)=0
$$

and by definition of the non-local action we get

$$
0=\partial_{\epsilon} \int_{c_{\mathcal{M} \epsilon}(\tilde{\Omega})} L\left(j\left(\Phi_{\epsilon}\right)\right) \mathrm{Vol}=\int_{\tilde{\Omega}} \partial_{\epsilon}\left[L\left(j\left(\Phi_{\epsilon}\right)\right) c_{M \epsilon}^{*} \mathrm{Vol}\right]
$$

for all $\tilde{\Omega} \subset \Omega$. This implies the local statement

$$
\partial_{\epsilon}\left[L\left(j\left(\Phi_{\epsilon}\right)\right) c_{M \epsilon}^{*} \mathrm{Vol}\right]=0
$$

On a manifold with a volume element the divergence can be written as

$$
\partial_{\epsilon}\left(c_{M \epsilon}^{*} \mathrm{Vol}\right)=\operatorname{div}\left(X_{M}\right) \mathrm{Vol} .
$$

With this definition and the chain rule we obtain equation 8

Conversely, if equation 8 is satisfied everywhere then the following is also true

$$
\left[\mathcal{D} L\left(X_{\mathcal{M}}\right)+\mathcal{D}_{\mathcal{V}} L\left(X_{Q}\right)+\mathcal{D}_{\mathcal{J}} L\left(D X_{Q}\right)+L \operatorname{div}\left(X_{\mathcal{M}}\right)\right]_{\left(\Phi_{\epsilon}\right)} c_{M \epsilon}^{*} \operatorname{Vol}=0
$$

where the equation in the brackets is to be taken at the point $\Phi_{\epsilon}$ for a small but finite $\epsilon$. Then the equation 10 is a differential of the equation 9 which we can write as

$$
\left.\partial_{\epsilon}\left[L\left(j\left(\Phi_{\epsilon}\right)\right) c_{M \epsilon}^{*} \mathrm{Vol}\right]\right|_{\epsilon \text { finite }}=0
$$

which leads to

$$
\partial_{\epsilon} \tilde{S}\left(\vec{\phi}_{\epsilon}\right)=0
$$

Integrating this equation from 0 to $\epsilon$ and using the fact that the $c_{0}=\mathbb{1}$, we get

$$
\tilde{S}\left(\vec{\phi}_{\epsilon}\right)=S(\vec{\phi})
$$

for a transformation $c_{\epsilon}$ sufficiently near the identity. However, since every connected one-dimensional subgroup is generated by a transformation of the form $c_{\epsilon}=\exp (\epsilon X)$ the above statement holds everywhere, which concludes the proof.

For local theories the corollary of the above theorem is known as the Noether theorem [1, 24]. In our case, however, it is valued on the product bundle $\mathcal{E}$ and it will now become apparent that the Noether theorem fails. 
Corollary 2. Let $X$ be a vector field on $\mathcal{E}$ with characteristic $X_{Q}$ and projection on the base manifold $X_{\mathcal{M}}$. Then $X$ induces a symmetry transformation iff

$$
\operatorname{div}\left(\mathcal{D}_{\mathcal{J}} L\left(X_{Q}\right)\right)+\operatorname{div}\left(L \cdot X_{\mathcal{M}}\right)+E\left(X_{Q}\right)=0 .
$$

With the Euler one form defined as above, $E:=\mathcal{D}_{\mathcal{V}} L-\operatorname{div}\left(\mathcal{D}_{\mathcal{J}} L\right)$.

Proof. From theorem 1, $X$ is a symmetry iff

$$
\left[\mathcal{D} L\left(X_{\mathcal{M}}\right)+\mathcal{D}_{\mathcal{V}} L\left(X_{Q}\right)+\mathcal{D}_{\mathcal{J}} L\left(\mathcal{D} X_{Q}\right)+L \operatorname{div}\left(X_{\mathcal{M}}\right)\right] \mathrm{Vol}=0 .
$$

By partial integration as described in section $\llbracket \mathrm{C}$ we obtain

$$
\operatorname{div}\left(\mathcal{D}_{\mathcal{J}} L\left(X_{Q}\right)\right)+\operatorname{div}\left(L \cdot X_{\mathcal{M}}\right)+\left[\mathcal{D}_{\mathcal{V}} L-\operatorname{div}\left(\mathcal{D}_{\mathcal{J}} L\right)\right]\left(X_{Q}\right)=0 \quad .
$$

In the local case the Euler one form coincides with the equations of motion Eq. 4] i.e. $E=E L$ and equation 11 becomes the well known Noether identity,

$$
\operatorname{div}\left(\mathcal{D}_{\mathcal{J}} L\left(X_{Q}\right)+L \cdot X_{\mathcal{M}}\right)+E L\left(X_{Q}\right)=0 .
$$

In the non local case, however, we have $E \neq E L$, which makes it difficult to connect it to physically relevant quantities and leads to the violation of the Noether theorem.

In general, equation 3 and 11 are very different, since the first one applies only on diagonal vector fields, whereas the second is true for a generic symmetry vector field on $T \mathcal{E}$, which in general does not even split into a direct sum of vector fields on separate vector bundles [26]. Such general vector fields, however, would correspond to non-local physical transformations where a shift of a field at a point $x$ depends on the field value at the point $y$. Only little is known about such vector fields and the corresponding symmetry transformations, and their relation to physics (in terms both of applicability and of meaning) is not clear. In the following we will therefore restrict ourselves to the case of diagonal symmetries, and present the relation between equations of motion and the symmetry condition in such cases. As we will see, even in the case of diagonal vector fields this relation is not trivial.

An additional remark is in order. Due to the mentioned problem of non-equivalent Lagrangians that differ by a total divergence on $\mathcal{M}$, the extension of the geometrical symmetry group to divergence symmetries can not be carried over from the local theory of variational problems. This extension is very important for a complete characterization of (local) symmetries of non-local actions, but goes beyond the scope of this paper (for further references see [24]).

\section{GENERALISED CONSERVATION LAW}

From the above treatment we see that, contrary to the local case, the equations of motion do not directly appear in the equation encoding the existence of a symmetry. This makes it difficult to combine these two in order to obtain physical conservation laws. In this section we introduce a possible connection of the two equations.

After briefly recalling the definition of the Fréchet derivative we show that it provides a natural connection between symmetries and equations of motion. This leads us to the generalization of the usual conservation law, which we state in theorem 2 ,

For the convenience of the reader we recall here the equations of motion and the symmetry equation, which we are going to combine in what follows

$$
E L(q)[X]=\sum_{i} \int_{\Omega} \delta^{i}(q) E^{i}[X] \operatorname{Vol} \quad \operatorname{div}\left(\mathcal{D}_{\mathcal{J}} L\left(X_{Q}\right)\right)+\operatorname{div}\left(L \cdot X_{\mathcal{M}}\right)+E\left(X_{Q}\right)=0 .
$$

We begin recalling the definition of the Fréchet derivative adopting the notation from [24]. We denote a set of smooth functions that depend on the base points, fields and their derivatives to some finite order by $\mathcal{A}$, and further denote a space of $l$-tuples of differential functions by $\mathcal{A}^{l}$, that is functions $P=\left(P^{1}, \cdots, P^{l}\right)$ where each $P^{i} \in \mathcal{A}$. The Fréchet derivative is defined as follows.

Definition 6. Let $P \in \mathcal{A}^{l}$ and $Q \in \mathcal{A}^{m}$ then the Fréchet derivative of $P$ in the direction $Q$ is a differential operator $D_{P}: \mathcal{A}^{m} \rightarrow \mathcal{A}^{l}$ defined as

$$
D_{P}(Q)=\left.\partial_{\epsilon}\right|_{0} P(q, \vec{\phi}+\epsilon Q(\vec{\phi}), \mathcal{D}(\vec{\phi}+\epsilon Q(\vec{\phi})), \cdots)
$$


We can now prove the following theorem.

Theorem 2. $X$ is a symmetry vector field of the non local action $S$ with the characteristic $Q$ if and only if

$$
E L\left(X_{Q}\right)(z)+\sum_{i=1}^{n} \operatorname{div}_{\mathfrak{D}^{i}}\left(A^{i}\right)(z)+\int_{\Omega} \operatorname{div}_{\mathcal{M}}(B) \delta^{\alpha}(z)+\int_{\Omega} \sum_{i=1}^{n} D_{L}\left(Q^{i}\right)\left[\delta^{\alpha}-\delta^{i}\right](z)=0,
$$

for all $\alpha \in\{1, \cdots, n\}$, with $A$ being a n-tuple

$$
A^{i}(z)=\int_{\Omega} D_{J^{i}} L\left(X^{i}\right) \delta^{i}(z)
$$

and $B \in T M$ as

$$
B=L \cdot X
$$

Before proving this theorem we first show the following trivial identity.

Lemma 3. The symmetry condition from the corollary 0 reads in terms of Fréchet derivatives of the Lagrangian as

$$
D_{L}\left(Q^{1}\right)+\cdots+D_{L}\left(Q^{n}\right)=-\operatorname{div}\left(L \cdot X_{M}\right) .
$$

where $X$ is the diagonal symmetry vector field, $Q^{i}$ the characteristic function of each individual component.

Proof. Let $Q$ denote the characteristic function of the symmetry vector field $X$. The Fréchet derivative of the Lagrangian in the direction of $Q$ is

$$
D_{L}(Q)=\operatorname{div}\left(D_{J} L\left(X_{Q}\right)\right)+E\left(X_{Q}\right) .
$$

Corollary 2 implies

$$
D_{L}(Q)+\operatorname{div}\left(L \cdot X_{M}\right)=0 .
$$

On the other hand, since the symmetry vector field is diagonal, we know that its characteristic vector field can be fibered as $X_{Q}=X_{Q}^{1}+\cdots+X_{Q}^{n}$ where $X_{Q}^{i}$ are the characteristic vector fields on the $i$ th factor of $\mathcal{E}$. Moreover they are all equal. Denote with $\tilde{Q}$ the characteristic of the single field $X_{Q}^{i}$ and write $Q=Q^{1}+Q^{2}+\cdots+Q^{n}$ with $Q^{i}=(0, \cdots, \tilde{Q}, \cdots 0)=\tilde{Q} \otimes e_{i}$. We then get

$$
D_{L}(Q)=D_{L}\left(Q^{1}\right)+\cdots+D_{L}\left(Q^{n}\right)
$$

Each $D_{L}\left(Q^{i}\right)$ is of the above form, namely

$$
D_{L}\left(Q^{i}\right)=\operatorname{div}_{\mathfrak{D}^{i}}\left(\mathcal{D}_{\mathcal{J}} L\left(X_{Q}^{i}\right)\right)+E\left(X_{Q}^{i}\right) .
$$

Inserting Eq17 in 16 proves the lemma.

We can now prove the main theorem.

Proof. Convoluting equation 18 with $\delta^{i}(z)$ we get

$$
\int_{\Omega} D_{L}\left(Q^{i}\right) \delta^{i}(z)=\int_{\Omega} \operatorname{div}\left(\mathcal{D}_{\mathcal{J}} L\left(X_{Q}^{i}\right)\right) \delta^{i}(z)+\int_{\Omega} E\left(X_{Q}^{i}\right) \delta^{i}(z)
$$

Due to Eq. 2 the only non vanishing divergence is on the subspace $\mathfrak{D}^{i} \subset \mathcal{M}$ on which the integrand is convoluted with $\delta^{i}(z)$ for $z \in M_{B}$. We therefore get

$$
\int_{\Omega} \operatorname{div}\left(D_{\mathcal{J}} L\left(X_{Q}^{i}\right)\right) \delta^{i}(z)=\operatorname{div}_{\mathfrak{D}^{i}}\left(A^{i}(z)\right) .
$$

The second term of equation 19 is a component of the Euler Lagrange equation applied on the vector field $X_{Q}^{i}$. By successive adding of equation 19 for all $i$ 's we obtain

$$
\sum_{i=1}^{N} \int_{\Omega} D_{L}\left(Q^{i}\right) \delta^{i}(z)=\sum_{i=1}^{N} \operatorname{div}_{\mathfrak{D}^{i}}\left(A^{i}(z)\right)+E L\left(X_{Q}\right)
$$


By lemma 3 we replace the left hand side by

$$
\sum_{i=1}^{N} \int_{\Omega} D_{L}\left(Q^{i}\right)\left[\delta^{i}(z)-\delta^{\alpha}(z)\right]-\int_{\Omega} \operatorname{div}\left(L \cdot X_{\mathcal{M}}\right) \delta^{\alpha}(z)=\sum_{i=1}^{N} \operatorname{div}_{\mathfrak{D}^{i}}\left(A^{i}\right)+E L\left(X_{Q}\right)
$$

where the term $\sum_{i=1}^{N} \operatorname{div}_{\mathfrak{D}^{i}}\left(A^{i}\right)$ is a sum of divergences on each individual domain $\mathfrak{D}^{i}$.

Conversely, if equation 14 holds then essentially following the previous steps backwards we arrive at

$$
\partial_{\epsilon} \int_{c_{\mathcal{M} \epsilon}(\Omega)} L\left(j\left(\Phi_{\epsilon}^{\alpha}\right)\right) \delta^{\alpha}(q) \mathrm{Vol}=0
$$

where $\Phi_{\epsilon}^{\alpha}$ is the section in which only the $\alpha$ 's field is transformed by $c_{\mathcal{V}} \circ \phi^{\alpha} \circ c_{\mathcal{M}}^{-1}$ and the rest $N-1$ fields are unchanged. By integration we get

$$
\partial_{\epsilon} \int_{\mathcal{C M}_{\epsilon}(\Omega)} L\left(j\left(\Phi_{\epsilon}^{\alpha}\right)\right) \mathrm{Vol}=0
$$

Since Eq. 14 holds for all $\alpha$ we get, for $c_{\epsilon}$ close to the identity,

$$
0=\int_{c_{\mathcal{M} \epsilon}(\Omega)} \sum_{\alpha=1}^{N} \partial_{\epsilon} L\left(j\left(\Phi_{\epsilon}^{\alpha}\right)\right) \mathrm{Vol}=\partial_{\epsilon} \int_{c_{\mathcal{M} \epsilon}(\Omega)} L\left(j\left(\Phi_{\epsilon}\right)\right) \mathrm{Vol}=\partial_{\epsilon} \tilde{S}\left(\vec{\phi}_{\epsilon}\right) .
$$

The usual group argument concludes the proof.

We define $\Delta^{\alpha}(z):=\sum_{i} \int_{\Omega} D_{L}\left(Q^{i}\right)\left[\delta^{i}-\delta^{\alpha}\right](z)$ and refer to it as to the non-local correction term (to the standard local conservation law). Theorem 2 states that the correction term is a divergence up to boundary terms

$$
\Delta^{\alpha}(z)-\operatorname{div}(A)(z)-\int_{\Omega} \operatorname{div}\left(L \cdot X_{M}\right) \delta^{\alpha}(z)=0 \quad .
$$

\section{Action with multiple kernels}

So far we have discussed the case in which the action is given by a single non-local Lagrangian. The usual action of non-local field theories is the combination of two or more terms with different type and combinatorics of non-localities. For instance the kinetic part is typically assumed to be local, whereas the interaction part can have non-localities and the various interaction terms have different types of them. As an example consider again a non-relativistic field theory of some atomic system, characterized by both a 2-body and a 3-body electromagnetic interaction with a Coulomb potential.

We can treat these cases by generalizing slightly our definition of the non-local action.

Definition 7. A non-local physical action with multiple kernels $S^{\text {mult }}[\phi]$ is a functional on $\Gamma(E)$, given by $\mathfrak{M}$ geometrical actions $S_{l}^{G}$, each of which is defined on the product bundle $\mathcal{E}_{l}=f_{l}^{*} E^{\times l}$, for $l \in\{1, \cdots, \mathfrak{M}\}$.

$$
S^{m u l t}[\phi]=\sum_{l=1}^{\mathfrak{M}} S_{l}^{G}\left[{ }_{l} \phi\right] .
$$

It is a straightforward calculation to check that the equations of motion are now given by

$$
\sum_{l} E L_{l}(q)[\cdot]=0,
$$

where $E L_{l}$ is the Euler Lagrange form for the $l$ th Lagrangian.

For the treatment of symmetries we again focus on the geometrical side, which is now the sum of individual functionals, each of which involve different domains of integration. It becomes difficult to formulate the most general geometrical transformation, since the tangent space on $\mathcal{E}_{l}$ is different from $\mathcal{E}_{m}$ if $l \neq m$. However, we can make sense of a symmetry transformation, if we again restrict ourselves only to diagonal vector fields, $X^{l}=(X, \cdots, X)$ with $X \in T E$. In this case we get 
Lemma 4. $X \in T E$ is a symmetry vector field of $\sum_{l=1}^{\mathfrak{M}} S_{l}^{G}$ iff it is a symmetry of each action $S_{l}^{G}$ separately That is $d S_{l}^{G}\left(X^{l}\right)=0$ for each $l \in\{1, \cdots, \mathfrak{M}\}$.

Proof. Let $c_{E \epsilon}^{l}$ and $c_{M \epsilon}^{l}$ be the flows of $X^{l}$. The variation of the generalised geometrical action is

$$
\partial_{\epsilon=0} \sum_{l=1}^{\mathfrak{M}} \tilde{S}_{l, \epsilon}^{G}\left(\phi_{\epsilon}\right)=0
$$

Each action contains an independent domain of integration. Since we defined the symmetry as a transformation that does not change the action for all subdomains, each term has to vanish separately. This can be seen in the following way. For $i \in\{1, \cdots, \mathfrak{M}\}$ the variation of the $i$ th action has to satisfy

$$
\mathrm{d} S_{\Omega_{i}}\left(X^{i}\right)=-\sum_{l \neq i}^{\mathfrak{M}} \mathrm{d} S_{\Omega_{l}}\left(X^{l}\right)
$$

Fixing $\Omega_{l \neq i}$, we get

$$
\mathrm{d} S_{\Omega_{i}}\left(X^{i}\right)=\text { const } \quad \forall \tilde{\Omega}_{i} \subset \Omega_{i} .
$$

Choosing $\Omega^{\prime \prime} \subset \Omega^{\prime} \subset \Omega_{i}$ and using the definition of the action we get

$$
\begin{aligned}
0 & =\mathrm{d} S_{\Omega^{\prime}}\left(X^{i}\right)-\mathrm{d} S_{\Omega^{\prime \prime}}\left(X^{i}\right)=\mathrm{d} S_{\Omega^{\prime \prime}}\left(X^{i}\right)+\mathrm{d} S_{\Omega^{\prime} \backslash \Omega^{\prime \prime}}\left(X^{i}\right)-\mathrm{d} S_{\Omega^{\prime \prime}}\left(X^{i}\right)= \\
& =\mathrm{d} S_{\Omega^{\prime} \backslash \Omega^{\prime \prime}}\left(X^{i}\right) .
\end{aligned}
$$

Taking $\tilde{\Omega} \subset \Omega^{\prime} \backslash \Omega^{\prime \prime} \subset \Omega_{i}$ leads to

$$
\mathrm{d} S_{\tilde{\Omega}}\left(X^{i}\right)=0
$$

which determines the constant. Applying the same reasoning for the remaining part $\sum_{l \neq i}^{\mathfrak{M}} \mathrm{d} S_{\Omega_{l}}\left(X^{l}\right)=0$ proves one direction of the lemma. The opposite direction is trivial.

For each action we get a symmetry equation of the form,

$$
E L_{l}\left[X_{Q}\right](q)+\sum_{i=1}^{N} \operatorname{div}_{\mathfrak{D}^{i}}\left(A^{i, l}\right)(q)_{l}+\int_{\Omega} \operatorname{div}_{\mathcal{M}}\left(B^{l}\right) \delta^{\alpha}(q)_{l}+\Delta^{\alpha, l}(q)=0 .
$$

The explicit form of $A^{i . l}, B^{l}$ and $\Delta^{\alpha, l}$ is given in the following theorem. Summing over $l$ we obtain the generalised conservation laws for the multiple action which leads us to our final result.

Theorem 3. Let $S$ be the non-local physical action given by a sum of geometrical actions as $\sum_{l=1}^{\mathfrak{M}} S_{l}^{G}$. Let $X^{l}=X^{\times l}$ be the symmetry of the lth geometrical action. The generalised conservation law for this symmetry take the form

$$
E L\left[X_{Q}\right](q)+\sum_{l=1}^{\mathfrak{M}}\left\{\sum_{i=1}^{N} \operatorname{div}_{\mathfrak{D}^{i}}\left(A^{i, l}\right)(q)_{l}+\int_{\Omega} \operatorname{div}_{\mathcal{M}}\left(B^{l}\right) \delta^{\alpha}(q)_{l}+\Delta^{\alpha, l}(q)\right\}=0
$$

with $A^{i, l}$ being

$$
A^{i, l}(q)=\int_{\Omega} \mathcal{D}_{\mathcal{J}^{i}} L^{l}\left[X^{i}\right] \delta^{i}(z) \operatorname{Vol}^{l}
$$

$B^{l} \in T M$ as

$$
B=L^{l} \cdot X^{l},
$$

and the non-local correction term

$$
\Delta^{\alpha, l}(z)=\sum_{i=1}^{l} \int_{\Omega} D_{L}\left(Q^{i}\right)\left[\delta^{i}-\delta^{\alpha}\right](z) V_{o l}^{l}
$$


For physical reasons we separate a special case as a corollary.

Corollary 3. Let the non-local physical action be composed of a local and a single non-local part, which we call the interaction. And let $X^{l o c}$ and $X^{\text {nloc }}=X^{\text {loc } \times N}$ be the symmetries of the local and the non-local geometrical action, respectively. The corresponding generalised conservation law reads

$$
\operatorname{div}_{M}\left(A^{l o c}+B^{l o c}\right)(q)+\sum_{i=1}^{N} \operatorname{div}_{\mathfrak{D}^{i}}\left(A^{i, i n t}\right)(q)+\int_{\Omega} \operatorname{div}_{\mathcal{M}}\left(B^{i n t}\right) \delta^{\alpha}(q)+\Delta^{\alpha, i n t}(q)=0
$$

with $A, B$ and $\Delta$ as above.

One sees that in this case the non-local correction originates only from the non-local interaction term. We will explicitly apply this result in the next section.

\section{On conserved charges}

In local field theories in which the base manifold is associated with space-time itself, one can integrate the conservation law over the spatial degrees of freedom. The resulting quantity is then a function of time only, the 'charge'. It is a consequence of the local conservation law that the charge is conserved, meaning that its time derivative vanishes. In the Hamiltonian language charges become the generators of the symmetry transformation, and might completely determine the dynamics of the system.

In more abstract field theories, such as group field theories, where the base manifold has no a priori relation to space-time, the notion of 'conserved' charges has no obvious counterpart. However, since the domain of the field in such theories is given by a direct product of manifolds $M^{1} \times \cdots \times M^{I}$ (such as space and time in non-relativistic case) we can define a generalised notion of charges as generators of the symmetry for translations along the single sub manifold $M^{i}$. We achieve this by integrating the generalised conservation law over the sub manifolds $M^{j \neq i}$. An appropriate notion of 'charge conservation' would be the vanishing of its covariant derivative on $M^{i}$. However, due to the non-local correction term $\Delta$, the generalised charge so defined will in general fail to be conserved. Nevertheless, in specific models in which the combinatorial structure of non-localities is such that the theory is local in $M^{i}$ (as it is the case, for example, for the time submanifold in non-relativistic field theories) the integral over the correction term $\Delta$ vanishes, as it can be easily verified, and the corresponding generalised charge is conserved.

The analysis of such generalised notion of conserved charges needs to be carried out in greater detail than we are doing here, as it has important consequences.

In space-time-based non-local field theories the notion of charges can help to define the Hamiltonian structure in the absence of a unique notion of time [3], but it may also simplify the equations of motion by reducing the degree of differential operators, as it does in local theories (see [24] for local examples).

In more abstract field theories the notion of charges will in general be very different from ordinary field theory due to the lack of direct physical interpretation of the background manifold. However, it can lead to useful insights both from the fundamental as well as from the computational point of view.

\section{PHYSICAL EXAMPLES}

We now apply our results to a non-local model of a complex scalar field with a non-local two-body interaction. Such model often appears in many-body quantum systems such as hydrodynamics, the theory of Bose-Einstein condensates or solid state physics. In this section we first introduce the action and the geometrical space according to our definitions. We then consider two physical symmetries and calculate the correspondent currents, together with their generalised conservation laws. Subsequently we show that for a particular choice of the interaction, the action can be rewritten as a local action, for which the usual Noether theorem holds. We conclude the section by comparing the currents of the local and non-local theories explicitly showing the appearance of the non-local correction term.

Our main purpose here is not to analyze the corresponding physics or explore the physical consequences of symmetries and their conservations laws for such systems. It is only to illustrate in some detail, in one specific example of a clear physical significance, the general formalism we have developed for carrying out such analysis in generic non-local field theories. 


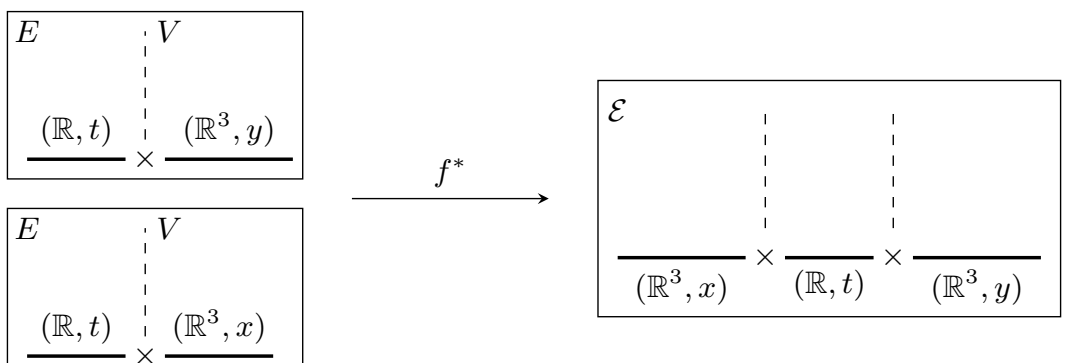

Figure 1: Bundle structure. Left hand side is the bundle with trivial combinatorics. The right hand side denote the combinatorics of the pull back bundle with the embedding map $f$ as in Eq. 1 Lower case letters denote the coordinate names.

\section{A. Non-local theory}

We consider a model given by a complex scalar field $\phi(t, x)$ and the non-local physical action

$$
\begin{aligned}
S & =\int \mathrm{d} t \mathrm{~d} x \phi^{*}(t, x) \partial_{t} \phi(t, x)+\nabla \phi^{*}(t, x) \cdot \nabla \phi(t, x) \\
& +\int \mathrm{d} t \mathrm{~d} x \mathrm{~d} y V(x, y)|\phi(t, x)|^{2}|\phi(t, y)|^{2} .
\end{aligned}
$$

We can think of this system as describing the hydrodynamic approximation of an atomic fluid. The local part of this model is the usual non-relativistic kinetic term. The non-local part describes the instantaneous interaction of two atoms at distinct space points. The nature of the interaction is determined by the potential term $V(x, y)$, which, for now, we leave unspecified except for the assumption that it vanishes sufficiently fast at infinity. The fields in such models are assumed to be normalized to the number of atoms in the location $x$. The density of such continuous system is defined by the modulus square of the field $\rho(t, x)=|\phi(t, x)|^{2}$.

The theory is local in time and non-local in space. The geometrical action is of the multi kernel type with the local and interaction part given by

$$
\begin{aligned}
& S^{1}=\int \mathrm{d} t \mathrm{~d} x \phi^{*}(t, x) \partial_{t} \phi(t, x)+\nabla \phi^{*}(t, x) \cdot \nabla \phi(t, x) \\
& S^{2}=\iint \mathrm{d} t \mathrm{~d} x \mathrm{~d} y V(x, y)|\phi(t, y)|^{2}|\phi(t, y)|^{2} .
\end{aligned}
$$

The bundle structure of $\mathcal{E}$ can be represented as in Figure 1 where the solid line denotes the base manifold and the dashed line refer to the fibers.

We use the following coordinates on the product jet bundle

$$
\mathcal{J}\left(E^{P}\right) \simeq \underbrace{\text { Base manifold }}_{(t, x, y)} \times \overbrace{\left(u, u^{*}\right)}^{\text {Fibers }} \times \overbrace{\left(v, v^{*}\right)}^{\mathbb{R}^{2} \times \mathbb{R}^{3}} \times \overbrace{\underbrace{\mathbb{R}^{2} \times \mathbb{R}^{6}}_{\left(u_{t} u_{t}^{*}, u_{x}, u_{x}^{*}\right)} \times \underbrace{\mathbb{R}^{2} \times \mathbb{R}^{6}}_{\left(v_{t}, v_{t}^{*}, v_{x}, v_{x}^{*}\right)}}^{\text {Jet spaces }} .
$$

In these coordinates the geometrical Lagrangians of $S^{1}$ and $S^{2}$ become

$$
\begin{aligned}
L^{\mathrm{loc}} & =u^{*} u_{t}+u_{x}^{*} u_{x} \\
L^{\text {nloc }} & =V(x, y)|u|^{2}|v|^{2} .
\end{aligned}
$$

1. $U(1)$ symmetry

We now consider the symmetry of the action under the phase rotation of the fields $\phi$. The geometrical action is symmetric under the transformation $c_{V \epsilon}(v)=e^{\imath \epsilon} v$ and $c_{M \epsilon}=\mathbb{1}$. Its infinitesimal generator is given by

$$
X=\imath \alpha v \partial_{v}-\imath \alpha v^{*} \partial_{v^{*}}
$$


which is already in its characteristic form. The characteristic function $Q$ is therefore $Q=\left(\imath \alpha v,-\imath \alpha v^{*}\right)$. Using this it is immediate to notice that the non-local correction term $\Delta^{1}$ to the conservation law vanishes

$$
\Delta^{1}=\partial_{\epsilon} V(x, y)|u|^{2}|v+\epsilon Q(v)|^{2}=V(x, y)|u|^{2}|v|^{2}(\imath \alpha-\imath \alpha)=0 \quad .
$$

We therefore obtain the continuity equation

$$
\operatorname{div}\left(J^{\text {loc }}\right)+\operatorname{div}\left(J^{\text {nloc }}\right)=0,
$$

where a priori one has an additional non-local contribution to the standard local one. However, since $D_{J} L^{\text {nloc }}=0$ as well as $X_{M}=0$, the non-local currents vanish, $J^{\text {nloc }}=0$, leading to the standard conservation law

$$
E L\left(X_{Q}\right)+\operatorname{div}\left(J^{\mathrm{loc}}\right)=0,
$$

with the well-known conserved current

$$
J^{\mathrm{loc}}=|u|^{2} \partial_{t}+\left(u_{x}^{*} u-u^{*} u_{x}\right) \partial_{x} \quad .
$$

Alternatively inserting the coordinates $u=\phi(t, x)$ the continuity equation on-shell gets the usual form

$$
\operatorname{div}\left(J^{\text {loc }}\right)=\partial_{t}\left(\phi^{*} \phi\right)+\operatorname{div}_{x}\left(\phi \nabla \phi^{*}-\phi^{*} \nabla \phi\right)=0,
$$

where $\nabla$ is the spatial gradient.

\section{Translation invariance}

If we assume that the potential $V$ depends only on the distance between the points $V(x, y)=V(|x-y|)$, the action also becomes translation invariant. That means that the geometrical action is invariant under a symmetry transformation with a vector field of the following form

$$
X=\mu^{t} \partial_{t}+\mu^{1} \partial_{x^{1}}+\mu^{2} \partial_{x^{2}}+\mu^{3} \partial_{x^{3}}=: \mu^{t} \partial_{t}+\vec{\mu} \cdot \partial_{x} \quad .
$$

The characteristic of the vector field has now two components

$$
Q^{2}=-\left(u_{t} \mu^{t}+u_{x} \cdot \vec{\mu}\right) \quad Q^{2 *}=-\left(v_{t}^{*} \mu^{t}+v_{x}^{*} \cdot \vec{\mu}\right),
$$

where we denote the scalar product between the 3 -dimensional jet coordinate $u_{x}$ and $\vec{\mu} \in \mathbb{R}^{3}$ with the dot. The Fréchet derivative is

$$
D_{L}\left(Q^{2}\right)=-V(x-y)|u|^{2}\left(\mu^{t}\left|v_{t}\right|^{2}+\vec{\mu} \cdot\left|v_{x}\right|^{2}\right) .
$$

The non-local correction term reads

$$
\Delta^{1}(t, z)=-\int_{\mathbb{R}^{3} \times \mathbb{R}^{3}} V(x-y)|u|^{2}\left(\mu^{t}\left|v_{t}\right|^{2}+\vec{\mu} \cdot\left|v_{x}\right|^{2}\right)[\delta(z-y)-\delta(z-x)] .
$$

By Corollary 3 , it follows that

$$
\operatorname{div}_{t, z}\left(T^{\mathrm{loc}} \cdot \mu\right)+\int \mathrm{d} x \operatorname{div}_{t, z, x}\left(L^{\mathrm{nloc}}(t, z, y) \cdot \mu\right)=\Delta^{1}(t, z) .
$$

Observing the $t, z$ divergence in the energy momentum tensor, we get

$$
\operatorname{div}_{t, z}(T \cdot \mu)=\Delta^{1}(t, z)-\int \mathrm{d} x \operatorname{div}_{x}\left(L^{\mathrm{nloc}}(t, z, y) \cdot \mu^{x}\right),
$$

with the energy-momentum tensor of the non-local theory given by

$$
T_{\mu \nu}(z)=T_{\mu \nu}^{\mathrm{loc}}(z)+\int_{\mathbb{R}^{3}} \mathrm{~d} y V(z-y) \rho(t, z) \rho(t, y) \delta_{\mu \nu} .
$$

By partial integration and again using the coordinates $u=\phi(t, x)$, we get 


$$
\begin{aligned}
\operatorname{div}(T \cdot \mu) & =\int \mathrm{d} x\left(\mu \cdot \nabla_{z}\right) V(z-x) \rho(t, z) \rho(t, x)-V(x-z) \rho(t, x)\left(\mu \cdot \nabla_{z}\right) \rho(t, z) \\
& +\int \mathrm{d} x V(z-x) \rho(t, z)\left(\mu_{t} \cdot \partial_{t}\right) \rho(t, x)-V(x-z) \rho(t, x)\left(\mu_{t} \cdot \partial_{t}\right) \rho(t, z)
\end{aligned}
$$

Note that, in the last equation, no boundary terms are present since they canceled by moving the gradient of the first term to the potential. Notice also that, if the potential $V$ is a contact interaction, that is if $V(x-y)=\delta(x-y)$, the interaction term becomes local and the terms on the right-hand-side of Eq. 22 cancel each other, leading to a conserved energy-momentum tensor.

Defining the mean value as $\langle\circ\rangle_{t}=\int \mathrm{d} x \circ \rho(t, x)$, we can rewrite equation 23 as

$$
\operatorname{div}(T \cdot \mu)=\rho(t, z) \mu \cdot \nabla_{t, z}\langle V\rangle(t, z)-\mu \cdot \nabla_{t, z} \rho(t, z)\langle V\rangle(t, z) \quad,
$$

which admits a nice physical interpretation. The divergence of the energy-momentum tensor is given by the mean force $\nabla_{t, x}\langle V\rangle$ due to non-local interactions and the dilution force $-\nabla_{t, z} \rho$ due to density fluctuations. Assuming a contact interaction, $V(x, y)=\delta(x-y)$, we obtain that the right-hand-side of the above equation cancels. In this case the mean force at a point has the same strength but the opposite direction as the dilution force. In the non-local case on the other hand the balance is broken since the fields at a distance induce an external mean field at each point turning the physical situation into that of a system in an external potential.

\section{B. Additional note: underlying local theory}

In this specific example, the non-local theory can be understood as an approximation of a local theory with additional degrees of freedom. We use this fact to give an interpretation for the additional terms in our generalised conservation law, as compared to the usual one. In fact, this is nothing else than a simpler case of the step from a theory of atoms interacting via a Coulomb potential to a description of the same atoms interacting via an electromagnetic field. Let us point out, however, that, on the one hand, we do not know if such rewriting of non-local field theories in terms of local ones is always possible, and that, on the other hand, our results concerning non-local theories do not rely on this possibility in any way.

We now present the local theory from which the above non-local emerges and show how the local, conserved currents relate to the non-local correction term. The Lagrangian of the local theory is given by

$$
L=\phi^{*} \partial_{t} \phi+\nabla \phi^{*} \cdot \nabla \phi+\alpha|\phi|^{2} A+\beta \nabla A \cdot \nabla A,
$$

with an auxiliary real scalar field $A$. The equation of motion for $A$ gives

$$
\Delta_{x} A(x, t)=\frac{\alpha}{2 \beta}|\phi|^{2}(x, t),
$$

which can be solved via the Green function

$$
G(x, y)=-\frac{1}{4 \pi|x-y|}
$$

We get

$$
A(x, t)=\frac{\alpha}{2 \beta} \int_{\mathbb{R}^{3}} \mathrm{~d} y \rho(t, y) G(y, x) .
$$

Inserting the solution into the Lagrangian Eq24, we get

$$
L=L^{\mathrm{loc}}+\frac{\alpha^{2}}{2 \beta} \int_{\mathbb{R}^{3}} \mathrm{~d} y \rho(t, x) \rho(t, y) G(x, y)+\beta \cdot \operatorname{div}(A \nabla A) \quad .
$$

Calling $V(x-y):=\frac{\alpha^{2}}{2 \beta} G(x, y)$, we get a non-local action from the form of Eq. 21] plus a total divergence, which does not change the equations of motion. The two actions are therefore equivalent.

Since the local and the non-local action are equivalent, we can calculate the currents of the local model and compare them to the non-local case. 
The $U(1)$ current of the local action is the same as for the non-local case since the real field $A$ does not change under the transformation $\phi \mapsto \phi_{\epsilon}=c_{E \epsilon} \circ \phi$. The energy-momentum tensor, however, is now conserved but depends on $A$.

$$
\operatorname{div}\left(T^{\phi} \cdot \mu+T^{A} \cdot \mu\right)=0
$$

The $A$-dependent part of the energy-momentum tensor reads

$$
\partial_{\mu} T_{\mu \nu}^{A} \mu^{\nu}=-\beta \partial_{i}\left(\partial^{i} A \partial_{\nu} A\right) \cdot \mu^{\nu}+\partial_{\mu}\left(L^{A} \cdot \delta_{\mu \nu}\right) \cdot \mu^{\nu}
$$

where we have explicitly written the divergence in components, using greek letters to range over $\left(t, x^{1}, x^{2}, x^{3}\right)$ and latin indices to range only over the spatial part.

Using the notation $\nu=(t, j)$, we calculate the first term $\partial_{i}\left(\partial^{i} A \partial_{\nu} A\right)$ as

$$
\begin{aligned}
\partial_{i}\left(\partial^{i} A \partial_{\nu} A\right) & =\Delta A \partial_{t} A-A \partial_{t} \Delta A+\Delta A \partial_{j} A-A \partial_{j} \Delta A+\partial^{i}\left(A \partial_{i} \partial_{t} A\right)+\partial^{i}\left(A \partial_{i} \partial_{j} A\right) \\
& =\frac{1}{\beta}\left[\int V(y, z) \rho(t, z) \partial_{t} \rho(t, y) \mathrm{d} y-\int V(y, z) \partial_{t} \rho(t, z) \rho(t, y) \mathrm{d} y\right] \\
& \frac{1}{\beta}\left[\int \partial_{j}^{z} V(z, y) \rho(t, z) \rho(t, y) \mathrm{d} y-\int V(y, z) \partial_{j}^{z} \rho(t, z) \rho(t, y) \mathrm{d} y\right] \\
& +\partial_{\mu}\left(A \partial_{\nu} \partial^{\mu} A\right) \quad,
\end{aligned}
$$

where the first equation follows from partial integration and the Schwarz theorem, used to interchange the derivatives. Inserting Eq28 into Eq27, we get the divergence of the energy-momentum tensor in the local theory

$$
\begin{aligned}
\operatorname{div}_{t, z}\left(T^{A} \cdot \mu\right) & =-\left[\int V(x, z) \rho(t, z)\left(\mu_{t} \cdot \partial_{t}\right) \rho(t, x) \mathrm{d} x-\int V(x, z)\left(\mu_{t} \cdot \partial_{t}\right) \rho(t, z) \rho(t, x) \mathrm{d} x\right] \\
& -\left[\int\left(\vec{\mu} \cdot \nabla_{z}\right) V(z, x) \rho(t, z) \rho(t, x) \mathrm{d} x-\int V(x, z)\left(\vec{\mu} \cdot \nabla_{z}\right) \rho(t, z) \rho(t, x) \mathrm{d} x\right] \\
& -\beta \cdot \operatorname{div}(A(\mu \cdot \nabla) \nabla A) \\
& +\partial_{\mu}\left(L^{A} \cdot \delta_{\mu \nu}\right) \cdot \mu^{\nu} .
\end{aligned}
$$

The last term can be put into the form

$$
\partial_{\mu}\left(L^{A} \cdot \delta_{\mu \nu}\right) \cdot \mu^{\nu}=\partial_{\mu}\left(\int \mathrm{d} y L^{\mathrm{nloc}}(z) \cdot \delta_{\mu \nu}\right) \cdot \mu^{\nu}+\partial_{\mu} \partial_{i}\left(A \partial^{i} A \cdot \delta_{\mu \nu} \mu^{\nu}\right)
$$

which is the $B=L^{\text {nloc }} \cdot X$ contribution to the energy momentum tensor. Rearranging the terms of Eq. 27 as follows we get

$$
\begin{aligned}
\operatorname{div}\left(T^{\phi} \cdot \mu+B\right) & =\left[\int\left(\vec{\mu} \cdot \nabla_{z}\right) V(z, x) \rho(t, z) \rho(t, x) \mathrm{d} x-\int V(x, z) \rho(t, x)\left(\vec{\mu} \cdot \nabla_{z}\right) \rho(t, z) \mathrm{d} x\right] \\
& +\left[\int V(x, z) \rho(t, z)\left(\mu_{t} \cdot \partial_{t}\right) \rho(t, x) \mathrm{d} x-\int V(x, z) \rho(t, x)\left(\mu_{t} \cdot \partial_{t}\right) \rho(t, z) \mathrm{d} x\right] \\
& +\beta \cdot \partial_{i}\left[\left(A\left(\mu^{\nu} \partial_{\nu}\right) \partial^{i} A\right)-\partial_{\mu}\left(A \partial^{i} A \cdot \delta_{\mu \nu} \mu^{\nu}\right)\right] .
\end{aligned}
$$

Compared to the generalised conservation law from the non-local theory, Eq23, stated here for convenience

$$
\begin{aligned}
\operatorname{div}_{t, z}(T \cdot \mu) & =\int \mathrm{d} x\left(\vec{\mu} \cdot \nabla_{z}\right) V(z-x) \rho(t, z) \rho(t, x)-V(x-z) \rho(t, x)\left(\vec{\mu} \cdot \nabla_{z}\right) \rho(t, z) \\
& +\int \mathrm{d} x V(z-x) \rho(t, z)\left(\mu_{t} \cdot \partial_{t}\right) \rho(t, x)-V(x-z) \rho(t, x)\left(\mu_{t} \cdot \partial_{t}\right) \rho(t, z),
\end{aligned}
$$

we see that the non-local correction comes from the auxiliary field $A$ and differs from a total divergence by an additional term

$$
\beta \cdot \partial_{i}\left[\left(A\left(\mu^{\nu} \partial_{\nu}\right) \partial^{i} A\right)-\partial_{\mu}\left(A \partial^{i} A \cdot \delta_{\mu \nu} \mu^{\nu}\right)\right]
$$




\section{WARD IDENTITIES FOR NON-LOCAL THEORIES}

In quantum field theory the symmetries of the classical action help to simplify the relations between correlation functions for the quantum fields. These simplified relations are called Ward identities. In the following we will review (in a sketchy fashion) the derivation of Ward identities in the functional integral formalism [27], and show that the classical results we derived above for non-local quantum field theories, imply relations between correlation functions just as in local quantum field theories.

The generating functional in Euclidean quantum field theories is defined in terms of the classical but possibly renormalized action $S$ as follows

$$
Z[J]=\int \mathcal{D} \phi e^{-S(\phi)+\int J \phi}
$$

Perform a coordinate transformation $\phi \mapsto \tilde{\phi}=\phi+Q$ where $Q$ is a characteristic of a symmetry vector field $X$ of the classical action $S$. Since we integrate over all fields the generating functional does not change and so we get

$$
\begin{aligned}
Z[J] & =\int \mathcal{D} \phi e^{-S(\phi)+\int J \phi} \\
& =\int \mathcal{D} \tilde{\phi} e^{-S(\tilde{\phi})+\int J \tilde{\phi}}=\tilde{Z}[J] \quad .
\end{aligned}
$$

Expressing $\tilde{Z}[J]$ in terms of the un-transformed fields $\phi$ we get to the first order in $Q$

$$
\tilde{Z}[J]=\int \mathcal{D} \phi\left[1+\operatorname{Tr}\left(D_{V} Q\right)\right] e^{-S(\phi)+\int J \phi+\mathrm{d} S(X)+\int J X_{Q}},
$$

where the term $\operatorname{Tr}\left(\mathcal{D}_{\mathcal{V}} Q\right)$ comes from the transformation of the functional measure. We also expanded the action to first order as $S(\tilde{\phi}) \approx S(\phi)+\mathrm{d} S\left(X_{Q}\right)$. Expanding the exponential to first order of $Q$ yields

$$
\tilde{Z}[J]=\int \mathcal{D} \phi e^{-S(\phi)+\int J \phi}\left[1+\operatorname{Tr}(\mathcal{D} \mathcal{V} Q)-\mathrm{d} S\left(X_{Q}\right)+\int J Q+\mathcal{O}\left(Q^{2}\right)\right]=Z[J]+\delta Z_{J}\left[X_{Q}\right]+\mathcal{O}\left(Q^{2}\right)
$$

where we define

$$
\delta Z_{J}\left[X_{Q}\right]=\left\langle\operatorname{Tr}\left(\mathcal{D}_{\mathcal{V} Q}\right)-\mathrm{d} S\left(X_{Q}\right)+\int J Q\right\rangle
$$

Using the notion of a functional average

$$
\langle\circ\rangle=\int \mathcal{D} \phi\left[e^{-S(\phi)+\int J \phi} \circ\right] .
$$

Equations 29 implies

$$
\delta Z_{J}\left[X_{Q}\right]=0
$$

Due to lemma 3, which stated $\mathrm{d} S\left(X_{Q}\right)=-\int_{\Omega} \operatorname{div}\left(L \cdot X_{\mathcal{M}}\right)$, we obtain

$$
\delta Z_{J}\left[X_{Q}\right]=\left\langle\operatorname{Tr}\left(\mathcal{D}_{\mathcal{V}} Q\right)+\int_{\Omega} \operatorname{div}\left(L \cdot X_{\mathcal{M}}\right)+\int J Q\right\rangle=0
$$

The anomaly term $\operatorname{tr}\left(\mathcal{D}_{\mathcal{V}} Q\right)$ comes from the transformation of the measure. If it does not vanish, the symmetries of the classical action do not imply a symmetry of the quantum effective action, and the symmetry is thus broken at the quantum level.

Equation 31 defines the Ward identities.

Expanding the generating functional in powers of $J$ Eq. 31 leads to the relation between coefficients of different powers of $J$, which correspond to different correlation functions.

So far, this statement is global, by which we mean that it depends on the whole domain $\Omega$. However, we can use our approach to the theorem 2 to obtain a semi-local statement.

To do this, we multiply the characteristic $Q$ of the symmetry vector field $X_{Q}$ by an arbitrary smooth function $\eta$ that vanishes on the boundaries of $U$. Then, we perform the variation of the action in the direction of $X_{Q \eta}$. Since the 
vector field $\imath_{N}\left(X_{Q \eta}\right)$ vanishes on the boundaries of $U$ the variation leads to the integral form of the Euler-Lagrange equations,

$$
\mathrm{d} S\left(X_{Q \eta}\right)=\sum_{i=1}^{N} \int_{\Omega} E_{i}\left[X_{Q}\right] \eta^{i} \mathrm{Vol}
$$

where $\eta^{i}$ denotes the smooth function $\eta$ defined over the $M^{i}=\operatorname{pr}^{i}(f(\mathcal{M}))$. Using Eq 18 this equation can be rewritten in terms of the Fréchet derivative as

$$
\mathrm{d} S\left(X_{Q \eta}\right)=\sum_{i=1} \int_{\Omega}\left[D_{L}\left(Q^{i}\right) \eta^{i}-\operatorname{div}\left(A^{i}\right) \eta^{i}\right]
$$

Due to lemma 3, which related the symmetries and Fréchet derivatives, we can instead write

$$
\mathrm{d} S\left(X_{Q \eta}\right)=\int_{\Omega}\left[\sum_{i=1} D_{L}\left(Q^{i}\right)\left(\eta^{i}-\eta^{\alpha}\right)\right]-\int_{U} \operatorname{div}(B) \eta^{\alpha}-\sum_{i=1}^{N} \int_{U} \operatorname{div}\left(A^{i}\right) \eta^{i}
$$

By the definition of the non-local correction term $\Delta^{\alpha}(z)=\int_{\Omega} \sum_{i=1}^{N} D_{L}\left(Q^{i}\right)\left[\delta^{i}-\delta^{\alpha}\right](z)$ we get

$$
\mathrm{d} S\left(X_{Q \eta}\right)=\int_{U}\left\{\Delta^{\alpha}(z)-\sum_{i=1}^{N} \operatorname{div}_{\mathcal{D}^{i}}\left(A^{i}\right) \delta^{i}(z)-\int_{U} \operatorname{div}_{M^{P}}(B) \delta^{\alpha}(z)\right\} \eta(z)
$$

which is a generalised version of the local equation $\mathrm{d} S=\int \operatorname{div}\left(J_{\text {phys }}\right) \cdot \eta$. Inserting this equation into Eq. 30, we obtain

$$
\int_{U} \eta(z)\left\langle\operatorname{Tr}\left(\mathcal{D}_{\mathcal{V}} Q\right)(z)-\operatorname{GCL}(z)+J Q(z)\right\rangle=0
$$

where GCL stays for the generalised Conservation Law. Since the above equation holds for all smooth functions $\eta$ on $U$. This leads to the modified local Ward identities

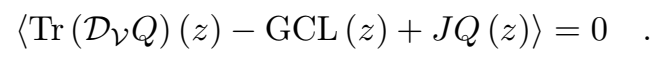

\section{CONCLUSIONS}

In this paper we have proposed a geometrical treatment of symmetries in non-local field theories, where the nonlocality is due to a lack of identification of field arguments in the action. We have shown that the existence of a symmetry of the action leads to a generalised conservation law, in which the usual conserved current acquires an additional non-local correction term, and thus obtained a generalization of the standard Noether theorem to such more exotic cases. We have illustrated the general formalism by discussing a specific physical example, in which this correction term can be interpreted as a dynamical external mean potential coming from the structure of the non-local field interaction.

Our analysis was focused on cases in which the geometrical Lagrangian is a function on the first jet bundle but we believe that an extension to higher jet bundles can be carried over from local theories without any complications. On the other hand, it is not clear whether the generalization to non-geometrical symmetries can be carried out in the same way as it is done in the local case. The major difficulty in this direction is the fact, that the total divergence on $\mathcal{M}$ can, in general, change the equations of motion leading to non-equivalent Lagrangians. A solution to this problem could lead to a full classification of (local) symmetries for non-local field theories.

We hope that our analysis and results will bring new insights in different areas of theoretical physics in which non-local field theories play a role, including condensed matter physics and cosmology. However, our main interest is the application of the above analysis to non-local models of quantum gravity, especially in the framework of group field theories (and thus, indirectly, of spin foam models and loop quantum gravity). The detailed analysis of known symmetries in group field theories and of their corresponding conservation laws is the subject of a follow-up paper. However, the interest of such analysis can already be envisaged. In particular, it will be important to develop the symmetry analysis of such models [22, 28] in a more systematic way and to use it to shed more light into their quantum geometric properties. The consequences of existing symmetries, encoded in the generalised Ward identities [29, 30], will also play an important role in the analysis of group field theory renormalization [31]. Finally, we expect symmetries and generalised conservation laws to be crucial for the further development and physical analysis of the effective cosmological dynamics extracted from group field theory models of quantum gravity [17]. 


\section{Acknowledgements}

We are very grateful to the members of the quantum gravity group at the AEI, and especially to Joseph Ben Geloun for several discussions and useful comments on this work.

[1] E. Noether, (1971) (translated in english 2005) arXiv:physics/0503066

[2] P. Kristensen and C. Møller, Dan. Mat. Fys. Medd. 27, no. 7 (1952)

[3] W. Pauli, IL Nuovo Cimento, vol 10, no. 5, pp 648-667 (1953)

[4] A. Leggett, "Quantum Liquids," Oxford University Press (2006); A. J. Leggett, Rev. Mod. Phys. 73, 307 (2001).

[5] S. Deser, R. Woodard, Phys.Rev.Lett.99:111301,2007, arXiv:0706.2151 [astro-ph]; S. Foffa, M. Maggiore, E. Mitsou, Int.J.Mod.Phys. A29 (2014) 1450116, arXiv:1311.3435 [hep-th]; T. Koivisto, Phys.Rev. D77 (2008) 123513, arXiv:0803.3399 [gr-qc]

[6] T. Banks, Nucl.Phys. B309 (1988) 493;

T. Banks, Phys.Scripta T117 (2005) 56-63, hep-th/0310288

C. Prescod-Weinstein, L. Smolin, Phys.Rev. D80 (2009) 063505, arXiv:0903.5303 [hep-th]

[7] S. Giddings, Phys.Rev. D74 (2006) 106005, hep-th/0605196

S. Giddings, Phys.Rev. D74 (2006) 106006, hep-th/0604072

P. Nicolini, arXiv:1202.2102 [hep-th]

[8] R. Sorkin, AIP Conf.Proc. 957 (2007) 142-153, arXiv:0710.1675] [gr-qc]

[9] G. Amelino-Camelia, L. Freidel, J. Kowalski-Glikman, L. Smolin, Phys.Rev. D84 (2011) 084010, arXiv:1101.0931 [hep-th]; S. Hossenfelder, Phys.Rev.Lett. 104 (2010) 140402, arXiv:1004.0418 [hep-ph]

[10] G. Gubitosi, F. Mercati, Class.Quant.Grav. 30 (2013) 145002, arXiv:1106.5710 [gr-qc];

S. Majid, Lect.Notes Phys. 541 (2000) 227-276, hep-th/0006166

[11] B. L. Hu, Int. J. Theor. Phys. 44, 1785 (2005) gr-qc/0503067;

T. Konopka, F. Markopoulou, S. Severini, Phys.Rev.D77:104029,200, arXiv:0801.086;

D. Oriti, PoS QG -PH (2007) 030 arXiv:0710.3276 [gr-qc]];

D. Oriti, Stud. Hist. Philos. Mod. Phys. 46 (2014) 186 arXiv:1302.2849 [physics.hist-ph]].

[12] D. Oriti, in Mathematical and Physical Aspects of Quantum Gravity, B. Fauser, et al. (eds) (Birkhaeuser, Basel, 2006), gr-qc/0512103;

A. Baratin and D. Oriti, J. Phys. Conf. Ser. 360, 012002 (2012) arXiv:1112.3270 [gr-qc]];

T. Krajewski, PoS QGQGS 2011, 005 (2011) arXiv:1210.6257 [gr-qc]];

D. Oriti, in The Planck Scale, J. Kowalski-Glikman, et al. (eds) AIP: conference proceedings (2009), arXiv:0912.2441 [hep-th];

D. Oriti, in: "Foundations of space and time", G. Ellis, J. Marugan, A. Weltman (eds.), Cambridge University Press (2012), arXiv:1110.5606 [hep-th]

[13] D. Oriti, arXiv:1310.7786 [gr-qc];

D. Oriti, in 'Loop Quantum Gravity', A. Ashtekar, J. Pullin (eds), World Scientific (to appear), arXiv:1408.7112 [gr-qc];

D. Oriti, J. P. Ryan and J. Thürigen, New J.Phys. 17 (2015) 2, 023042 arXiv:1409.3150 [gr-qc].

[14] A. Ashtekar and J. Lewandowski, Class. Quant. Grav. 21, R53 (2004) gr-qc/0404018];

T. Thiemann, "Modern canonical quantum general relativity", Cambridge, UK: Cambridge Univ. Pr. (2007) 819p. C. Rovelli, PoS QGQGS 2011, 003 (2011) arXiv:1102.3660 [gr-qc]].

[15] R. Gurau and J. P. Ryan, SIGMA 8 (2012) 020 arXiv:1109.4812 [hep-th]];

V. Rivasseau, Fortsch.Phys. 62 (2014) 81-107, arXiv:1311.1461 [hep-th]

[16] M. Bojowald, Rept.Prog.Phys. 78 (2015) 023901, arXiv:1501.04899 [gr-qc]

[17] S. Gielen, D. Oriti and L. Sindoni, Phys. Rev. Lett. 111 (2013) 031301 arXiv:1303.3576 [gr-qc]];

S. Gielen, D. Oriti and L. Sindoni, JHEP 1406 (2014) 013, arXiv:1311.1238 [gr-qc];

S. Gielen, Class.Quant.Grav. 31 (2014) 155009, arXiv:1404.2944 [gr-qc]; G. Calcagni, Phys. Rev. D 90 (2014) 064047, arXiv:1407.8166 gr-qc];

S. Gielen and D. Oriti, New J. Phys. 16, 123004 (2014) arXiv:1407.8167 [gr-qc]]; S. Gielen, Phys.Rev. D91 (2015) 4, 043526, arXiv:1411.1077] [gr-qc];

L. Sindoni, arXiv:1408.3095 [gr-qc];

S. Gielen, arXiv:1505.07479 [gr-qc];

D. Oriti, D. Pranzetti, J. Ryan, L. Sindoni, arXiv:1501.00936 [gr-qc]

[18] C. Bloch, Mat. Fys. Medd. XXVI, nr. 1 (1950)

[19] R. Marnelius, Phys. Rev. D 8, 2472 (1973)

[20] Z. Huang, (2012), arXiv:1203.1149 [math-ph]

[21] H. Yukawa, Phys. Rev. 77, 219 (1950)

[22] J. Ben Geloun, J.Math.Phys. 53 (2012) 022901, arXiv:1107.3122 [hep-th]

[23] R. Abraham and J. E. Marsden, "Foundations of Mechanics" (second edition), AMS Chelsea (2008) 
[24] P. J. Olver, "Applications of Lie Groups to Differential Equations" (second edition), Springer (1998)

[25] M. Giaquinta and S. Hildebrandt, Calculus of Vatiations I, Springer, (1996)

[26] S. Kurz, (2011) arXiv:1106.0926v1

[27] V. P. Nair, "Quantum Field Theory A Modern Perspective", Springer (2004)

[28] A. Baratin, F. Girelli, D. Oriti, Phys.Rev. D83 (2011) 104051, arXiv:1101.0590 [hep-th]

[29] J. Ben Geloun, J.Phys. A44 (2011) 415402, arXiv:1106.1847] [hep-th]

[30] D. O. Samary, C. I. Pérez-Sánchez, F. Vignes-Tourneret and R. Wulkenhaar, arXiv:1411.7213 [hep-th]; V. Lahoche, D. Oriti, V. Rivasseau, JHEP 1504 (2015) 095, arXiv:1501.02086 [hep-th]

[31] J. Ben Geloun and V. Rivasseau, Commun. Math. Phys. 318, 69 (2013) arXiv:1111.4997 [hep-th]];

S. Carrozza, D. Oriti and V. Rivasseau, Commun. Math. Phys. 327, 603 (2014) arXiv:1207.6734 [hep-th]];

D. O. Samary and F. Vignes-Tourneret, Commun. Math. Phys. 329, 545 (2014) arXiv:1211.2618 [hep-th]];

S. Carrozza, D. Oriti and V. Rivasseau, Commun. Math. Phys. 330, 581 (2014) arXiv:1303.6772 [hep-th]);

J. Ben Geloun, Commun. Math. Phys. 332, 117-188 (2014) arXiv:1306.1201 [hep-th];

J. Ben Geloun, "On the finite amplitudes for open graphs in Abelian dynamical colored Boulatov-Ooguri models," J. Phys. A 46, 402002 (2013) arXiv:1307.8299 [hep-th]];

S. Carrozza, Springer Theses, 2014 (Springer, NY, 2014), arXiv:1310.3736 [hep-th];

J. Ben Geloun, Class. Quant. Grav. 29, 235011 (2012) arXiv:1205.5513 [hep-th]];

D. O. Samary, "Beta functions of $U(1)^{d}$ gauge invariant just renormalizable tensor models," Phys. Rev. D 88, 105003 (2013) arXiv:1303.7256 [hep-th]];

S. Carrozza, "Discrete Renormalization Group for SU(2) Tensorial Group Field Theory," Ann. Inst. Henri Poincaré Comb. Phys. Interact. 2 (2015), 49-112, arXiv:1407.4615 [hep-th];

S. Carrozza, Phys. Rev. D 91, 065023 (2015), arXiv:1411.5385 [hep-th] 